\title{
Photoelectron Flux Buildup in the Plasmasphere
}

\author{
George P. Mantas and Herbert C. Carlson \\ The University of Texas at Dallas, Richardson, Texas 75080 \\ VINCENT B. WICKWAR \\ Stanford Research Institute, Menlo Park, California 94025
}

\begin{abstract}
Processes which confine photoelectrons to the plasmasphere (e.g., collisional backscattering from the thermosphere and magnetic trapping due to pitch angle redistribution through Coulomb collisions in the plasmasphere) tend to increase the steady state photoelectron flux in the plasmasphere above the amplitude level that would otherwise have been attained. Theoretical calculations are presented of steady state photoelectron fluxes in the plasmasphere, for specified atmospheric and ionospheric conditions. (Observational plasma line intensity data for these conditions exist and will be compared elsewhere.) General features of the angular distribution are presented and compared with observations. The transparency of the plasmasphere and the backscattering properties of the thermosphere are investigated. The buildup effect due to collisional backscatter alone is calculated, and the combined buildup effect of pitch angle diffusion and backscatter is estimated. It is found that the inclusion of these effects increases the steady state photoelectron flux amplitude in the plasmasphere by about $50 \%$ over the value obtained when the buildup effects are neglected. The calculated steady state photoelectron fluxes in the plasmasphere are in good agreement with the available observations.
\end{abstract}

\section{INTRODUCTION}

In his study of the thermal structure of the ionosphere, Hanson [1963] showed that a substantial fraction of the photoelectrons produced around $300 \mathrm{~km}$ escape to the plasmasphere and thereby affect the electron gas heating rate above this altitude. Following experimental verification of this prediction [Carlson, 1966], several calculations of the photoelectron 'escape' flux have been presented [Nisbet, 1968; Nagy and Banks, 1970; Cicerone and Bowhill, 1971b; Mantas and Bowhill, 1975]. The problem of photoelectron escape has also been studied in connection with the thermal structure of the ionosphere and the plasmasphere [Geisler and Bowhill, 1965a, b; Evans, 1967a, b. 1968; Sanatani and Hanson, 1970; Bauer et al.. 1970; Swartz et al., 1975; Bailey et al., 1975], the predawn electron temperature and the $6300-\AA$ airglow enhancement [Carlson, 1966, 1967, 1974; Carlson and Weill, 1967; Fontheim et al., 1968; Noxon and Johanson, 1970; Nagy and Banks, 1971; Wickwar. 1972, 1974], and possible perturbations on the predawn electron concentration [Shawhan et al., 1970; Nagy et al., 1973]. Incoherent scatter radar plasma line intensity observations [Ynguesson and Perkins, 1968; Cicerone and Bowhill, 1971a] and in situ measurements by satellite-borne probes [Rao and Donley, 1969; Rao and Maier, 1970; Knudsen, 1972; Hays and Sharp, 1973; Doering et al., 1970, 1976] have provided valuable information about the steady state flux amplitude and its energy and pitch angle distribution. Despite this substantial progress, there remain important aspects of the steady state photoelectron flux which have not been adequately investigated. The work presented herein is directed toward the investigation of certain such attributes of the photoelectron flux at high altitudes.

The theoretical problem under consideration is the determination of the photoelectron distribution in a magnetic field tube in the ionosphere above $\sim 120 \mathrm{~km}$. For a number of physical and computational reasons it has been found to be convenient, however, to calculate the photoelectron distribution in the ionosphere below a certain altitude (usually taken

Copyright (C) 1978 by the American Geophysical Union. at $1000 \mathrm{~km}$ ) separately from that in the plasmasphere above. This separation necessitates the imposition of a boundary condition (the downward photoelectron flux) at this altitude, which is not known, however, but must be found along with the photoelectron distribution in the ionosphere and the plasmasphere. It also naturally leads to terminology as 'photoelectron escape,' 'escape flux,' etc., which, although it is useful, conceals the essential ionosphere-plasmasphere coupling and, as a consequence, can be misleading; indeed, it has been the source of errors. (Here we are again faced with the not so uncommon situation in which originally useful concepts eventually become sources of error and a hindrance to the further development of the theory.) If the incoming flux (upper boundary condition) is assumed to be zero and the upward moving electrons are assumed to be free to escape, then the upward flux at the upper 'boundary' can be properly called escape flux. The incoming flux at the boundary is not, however, zero because a fraction of the electrons passing through this boundary in the outward direction reenter the ionosphere after they are backscattered from the conjugate ionosphere [Banks and Nagy, 1970; Nagy and Banks, 1970; Mantas and Walker, 1976] and the plasmasphere [Lejeune and Wormser, 1976].

The backscattering of electrons from the thermosphere and the redistribution of the pitch angle through Coulomb collisions in the plasmasphere imply that photoelectrons can be quasi-trapped in the plasmasphere and, as a consequence, that the steady state photoelectron flux in a magnetic field tube in the plasmasphere can be much larger than the sum of the fluxes entering the field tube boundaries. This has been pointed out by Sanatani and Hanson [1970] and Nagy and Banks [1970] and is consistent with the observed large thermal electron gas temperature gradients at high altitudes in the mid-latitude ionosphere [Evans, 1967a, b; Petit, 1968; Hagen and Hsu, 1974], which cannot be explained on the basis of the simple photoelectron escape concept alone [Evans and Mantas, 1968; Evans, 1968; Sanatani and Hanson, 1970]. That quasi-trapping of photoelectrons in the plasmasphere occurs has also been inferred by Wrenn [1974] from Isis satellite measurements and 
theoretically demonstrated by Lejeune and Wormser [1976]. Therefore in the actual physical situation the photoelectron flux observed at the ionosphere-plasmasphere boundary represents essentially the steady state rate of photoelectron exchange between the ionosphere and the plasmasphere, and not an escape flux in the sense that this term has hitherto been used. It is therefore clear that to calculate the actual photoelectron flux at any point on a magnetic field line in the ionosphere or the plasmasphere, one must obtain a solution of the photoelectron transport equation either by treating the entire magnetic field tube as a single unit or by properly matching at the boundary separate solutions for the two regions. In the present calculations the latter approach, with certain clearly stated simplifications, is taken.

\section{General Considerations Regarding Photoelectrons IN THE IONOSPHERE AND Plasmasphere}

Before we proceed further, it will be useful to consider the exchange of photoelectrons between the ionosphere and the plasmasphere and the mean photoelectron lifetimes in the ionosphere and plasmasphere at large. For the present purpose we will define the photoelectron lifetime in the ionosphere as the mean time a freshly produced photoelectron of average energy (about $30 \mathrm{eV}$ ) remains in the ionosphere before it either is thermalized or escapes to the plasmasphere. It is then clear that for given photoelectron production conditions those factors which increase the photoelectron lifetime in a certain region of the ionosphere lead to increases in the steady state number of photoelectrons, and in the photoelectron flux amplitude, in that region.

The steady state photoelectron density within a certain volume in the ionosphere is determined by the rates of photoelectron production and loss. For the ionosphere below $1000 \mathrm{~km}$, as a whole, photo-ionization and secondary ionization constitute the major photoelectron sources. To these sources, one must also add whatever photoelectrons may enter the ionosphere from above. Photoelectron losses consist of photoelectron thermalization (through inelastic collisions with the neutral atmospheric constituents and through 'continuous' energy transfer to the ambient electron gas) and photoelectron escape to the plasmasphere. For any small volume element within the ionosphere, transport serves as both a photoelectron source and a sink. In viewing the ionosphere as a whole, apart from leading to photoelectron escape to the plasmasphere, photoelectron transport redistributes the photoelectron population in altitude, from regions of high thermalization rate to regions of low thermalization rate and thereby affects the mean photoelectron lifetime.

Similar factors also determine the steady state number of photoelectrons in a magnetic field tube in the plasmasphere. In this case the photoelectron escape flux from the ionosphere constitutes the photoelectron source (possible energization of thermal electrons through wave particle interactions or other means being neglected). Photoelectron thermalization through energy loss to the ambient thermal plasma and escape to the ionosphere from the field tube boundaries constitute the photoelectron losses in the plasmasphere.

The thermalization time for an energetic electron of initial energy $E_{0}(\mathrm{eV})$ in a completely ionized plasma of uniform density $N_{e}\left(\mathrm{~cm}^{-3}\right)$ and temperature of a few thousand kelvin is approximately given by $\tau(s) \simeq 4300\left(E_{0}{ }^{3 / 2} / N_{e}\right)$. By taking the mean thermal electron density in a magnetospheric field tube as $5 \times 10^{3} \mathrm{~cm}^{-3}$ the thermalization time for a $20-\mathrm{eV}$ photoelectron (approximately equal to the mean energy of the escape flux [see Mantas and Bowhill, 1975]) confined in the plasmasphere is about $80 \mathrm{~s}$. In this time interval the photoelectron will cover a distance $\Delta s \simeq 2 \times 10^{11} \times\left(E_{0}^{2} / N_{e}\right) \simeq 1.5 \times 10^{10} \mathrm{~cm}$. The path length of an electron entering a field tube with $L=$ 1.5 at $1000 \mathrm{~km}$, with a pitch angle of, say, $87^{\circ}$, through the magnetosphere to the magnetic conjugate is about $1.73 \times$ $10^{\circ} \mathrm{cm}$ (collisions neglected) and decreases by about a factor of 2 as the initial pitch angle (measured from the forward direction) goes to zero. Therefore an average photoelectron escaping from the ionosphere over Arecibo $(L=1.42)$, if it could be contained in the plasmasphere, has sufficient energy to traverse the plasmasphere 10-20 times (depending on its initial pitch angle) before being thermalized.

In this estimate we have considered photoelectrons entering a plasmasphere field tube from the ionosphere below. If pitch angle scattering in the plasmasphere and collisional backscattering of the phtoelectrons at the field tube boundaries are neglected, then the photoelectrons will traverse the plasmasphere only once and will be subsequently absorbed in the ionosphere. In such a case the photoelectron lifetime in the plasmasphere will be equal to the time required for a single traversal of the plasmasphere rather than equal to the thermalization time estimated above. Essentially, the assumption of a single traversal is implicitly made when one estimates the photoelectron flux in the plasmasphere as the sum of the fluxes entering the field tube boundaries from the ionosphere below. However, collisional backscattering of photoelectrons from the thermosphere and pitch angle scattering in the plasmasphere constitute mechanisms of containment of photoelectrons in the plasmasphere. The mean photoelectron lifetime in the plasmasphere is therefore higher than the time required for a single traversal of the distance between the field tube boundaries, and, as a consequence, the steady state photoelectron density and flux in the plasmasphere are higher than the corresponding quantities when containment effects are neglected.

To obtain a quantitative estimate of the photoelectron flux buildup in the plasmasphere due to collisional backscatter containment alone, we follow a procedure suggested by Mantas [1973, 1975]. The photoelectron transport equation (see (1) below) is solved in the ionosphere below $1000 \mathrm{~km}$ for sunlit conditions and an upper boundary condition of no incoming flux. This solution provides the first estimate of the steady state flux in the ionosphere and of the escape photoelectron flux at $1000 \mathrm{~km}$. The attenuation of the escape flux in traversing the plasmasphere is then calculated, and (1) is again solved for the ionosphere below $1000 \mathrm{~km}$, with the primary photoelectron production rate set equal to zero and an incoming flux (upper boundary condition) equal to the attenuated escape flux. This solution provides the first estimate of the electron 'albedo' and the contribution to the steady state flux below $1000 \mathrm{~km}$ by photoelectrons originating in the conjugate ionosphere. Further contributions due to multiple reflections between the conjugate ionospheres are obtained by repeating the last cycle.

The linearity of (1) permits the construction of the steady state photoelectron fluxes in the ionosphere and the plasmasphere by superposition of the appropriate components of these solutions. In this procedure, pitch angle scattering in the plasmasphere is neglected. To obtain an estimate of the steady state flux in the plasmasphere that includes collisional backscatter from the thermosphere and pitch angle scattering in the plasmasphere, the results of the above calculations are combined with those of Lejeune and Wormser [1976] which include pitch angle diffusion in the plasmasphere but not backscatter from the thermosphere. 


\section{TheORY OF Photoelectron Thermalization AND TRANSPORT IN THE IONOSPHERE}

The number of photoelectrons found at any time in a volume element at a certain altitude, with energies in a specified energy interval and velocity vectors in a specified solid angle element, is determined by the balance between production and loss processes. These include photo-ionization and secondary ionization by electron impact, discrete and continuous energy degradation, and transport. If it is assumed that (1) photoelectron thermal-electron interactions lead to continuous energy transfer but do not affect the direction of the photoelectron motion, (2) elastic collisions with neutrals and ions lead to photoelectron scattering without energy transfer, (3) inelastic collisions lead to discrete energy transfer and forward scattering, (4) ionizing collisions lead to single ionization and forward scattering, and (5) transport occurs only along the magnetic field (which is assumed to be uniform), it can then be shown [see Mantas, 1975] that the steady state photoelectron flux $\Phi(z, E, \mu)\left(\mathrm{cm}^{-2} \mathrm{eV}^{-1} \mathrm{~s}^{-1} \mathrm{sr}^{-1}\right)$ satisfies the equation

$$
\begin{aligned}
& \left\{\mu \frac{\partial}{\partial z}-\left(\beta N_{e}(z) / E\right) \frac{\partial}{\partial E}+\left(\beta N_{e}(z) / 2 E^{2}\right)\right. \\
& \left.\quad+\sum_{l} N_{l}(z) \sigma_{l}^{\tau}(E)\right\} \Phi(z, E, \mu)=Q(z, E, \mu) \\
& \quad+2 \pi \sum_{l} N_{l}(z) \sigma_{l}^{e}(E) \int_{-1}^{1} P_{l}^{e}\left(E, \mu, \mu^{\prime}\right) \Phi\left(z, E, \mu^{\prime}\right) d \mu^{\prime} \\
& \quad+\sum_{l} \sum_{j} N_{l}(z) \sigma_{l}^{j}\left(E+W_{l j}\right) \Phi\left(z, E+W_{l j}, \mu\right) \\
& \quad+\sum_{l} N_{l}(z) \int \sigma_{l}^{\prime}\left(E^{\prime}\right) P_{l}^{\prime}\left(E^{\prime}, E\right) \Phi\left(z, E^{\prime}, \mu\right) d E^{\prime}
\end{aligned}
$$

where $z, E$, and $\mu$ are independent variables representing the distance along the magnetic field lines in centimeters, the energy in electron volts, and the cosine of the pitch angle (measured from the upward direction), respectively; $N_{e}(z), N_{l}(z)$ are the electron, neutral, and ion number densities in cubic centimeters; $l$ is the ionosphere composition index that includes all ionospheric constituents with the exception of thermal electrons; $\sigma_{l}{ }^{\top}(E), \sigma_{l}{ }^{e}(E), \sigma_{l}{ }^{J}(E)$, and $\sigma_{l}{ }^{l}(E)$ are the total, elastic, excitation, and ionization cross sections in square centimeters, respectively; $W_{l j}$ is the excitation potential of the $j$ state of the constituent $l ; P_{l}^{e}\left(E, \mu^{\prime}, \mu\right)$ is the angular distribution density for elastic scattering of electrons with pitch angle cosines in the interval $d \mu^{\prime}$ about $\mu^{\prime}$ to $d \mu$ about $\mu ; P_{l}^{\prime}\left(E, E^{\prime}\right)$ is the energy distribution density of the postcollision electrons in ionizing collisions. For any fixed value of $E^{\prime} \geq W_{l i}$ (where $W_{l t}$ is the ionization potential of the state $i$ of the constituent $l$, and $E^{\prime}$ is the incident electron energy) the integral

$$
\int_{0}^{E^{\prime}-W_{\prime}} P_{l} l\left(E, E^{\prime}\right) d \dot{E}=2
$$

in accordance with the assumption that only single ionization occurs. $Q(z, E, \mu)\left(\mathrm{cm}^{-3} \mathrm{eV}^{-1} \mathrm{~s}^{-1} \mathrm{sr}^{-1}\right)$ is the photoelectron source, and $\beta=2.59 \times 10^{-12} \mathrm{eV}^{2} \mathrm{~cm}^{2}$.

The photoelectron source can be written as $Q(z, E, \mu)=q(z$, $E) g(E, \mu)$, where $q(z, E)$ is the photo-ionization rate energy spectrum and

$$
g(E, \mu)=\frac{1}{2}\left\{1-[\alpha(E) / 2] P_{2}(\cos \gamma) P_{2}(\mu)\right\}
$$

is the pitch angle distribution of the source electrons [Manson et al., 1974]. The cosine of the angle $\gamma$ between the incident photon and the geomagnetic field is given by [Mariani, 1964]

$$
\cos \gamma=\cos \delta \sin (I+\lambda) \cos \phi-\sin \delta \cos (I+\lambda)
$$

Here, $\delta$ is the solar declination, $I$ is the magnetic dip angle, $\lambda$ is the geographic latitude, and $\phi$ is the local time angle; $\alpha(E)$ is an energy dependent asymmetry parameter [cf. Manson et al., 1974], and $P_{2}(x)=\frac{1}{2}\left(3 x^{2}-1\right)$ is recognized as the seconddegree Legendre polynomial.

The second and third terms in (1) arise from the photoelectron thermal-electron interaction [Mantas, 1975]. The energy loss per unit length along the photoelectron path, for $E>2$ $\mathrm{eV}$, by this process is approximately given [cf. Schunk and Hays, 1971] by

$$
d E / d s=-\left(\beta N_{e}(s) / E\right)(\mathrm{eV} / \mathrm{cm})
$$

For $E<2 \mathrm{eV}$ the energy transfer rate is strongly dependent upon the ambient electron temperature, and (1) and (2) are no longer valid.

A numerical method for solving (1) has been discussed by Mantas [1975], and solutions for a sunlit ionosphere without contribution from the conjugate ionosphere have been given by Mantas and Bowhill [1975]. This equation has also been applied to the problem of soft electron penetration into the atmosphere by Mantas and Walker [1976]. The present calculations are essentially similar to those in the above references except for the inclusion of a small term in the difference form of (1) given by Mantas [1975], neglected in the first of the above references, and the use of boundary conditions appropriate for the present problem.

\section{Photoelectron Fluxes in the Plasmasphere}

A proper treatment of the problem of photoelectron fiuxes in the plasmasphere would require the solution of the transport equation for photoelectrons that takes account of the divergence of the magnetic field lines and includes the effects of collisions between photoelectrons and the ambient plasma particles. In the present treatment we take account of the divergence of the magnetic field lines and its effect on the photoelectron energy loss to the ambient thermal electron gas. However, we neglect pitch angle redistribution through collisions in the plasmasphere. Quantitative estimates of the effect of pitch angle diffusion through collisions in the plasmasphere on the magnitude of the flux buildup will be made later. However, we qualitatively note here that in addition to injecting a fraction of photoelectrons into magnetically trapped orbits, collisions inhibit transport (the same effect as that found in the ionosphere, which has been discussed by Banks and Nagy [1970] and Mantas and Bowhill [1975]). Both effects tend to retain a larger fraction of photoelectrons in the plasmasphere than when they are neglected. Inclusion of collisions would therefore lead to higher steady state photoelectron fluxes in the plasmasphere than when they are neglected. The importance of collisions in retaining a substantial number of photoelectrons in the plasmasphere has been brought out by the work of Wormser [1973].

A photoelectron traversing the plasmasphere interacts with the ambient thermal electron gas with an energy tranfer rate given by (2). Integration of (2) gives

$$
E(s)=\left\{E^{2}\left(s_{0}\right)-2 \beta \int_{s_{0}}^{s} N_{e}\left(s^{\prime}\right) d s^{\prime}\right\}^{1 / 2}
$$

for the energy of a photoelectron at a distance $\Delta s$ from its initial position $s_{0}$, where it had the energy $E\left(s_{0}\right)$. The integra- 
tion in (3) must be carried out along the photoelectron trajectory.

When collisions are neglected, the distance element $d s^{\prime}$ along the path of an electron moving in a dipole field can be easily obtained from the equations of the magnetic field lines and the constraint imposed by the transverse adiabatic invariant as

$$
\begin{aligned}
d s^{\prime}= & R_{E} L \sin ^{4} \theta\left(3 \cos ^{2} \theta+1\right)^{1 / 2} d \theta\left\{\left[\sin ^{6} \theta-\sin ^{2} \alpha_{0}\right.\right. \\
& \left.\left.\cdot \sin ^{6} \theta_{0}\left(\left(3 \cos ^{2} \theta+1\right) /\left(3 \cos ^{2} \theta_{0}+1\right)\right)^{1 / 2}\right]^{1 / 2}\right\}^{-1}
\end{aligned}
$$

where $R_{E}$ is the earth's radius, $L$ is the McIlwain coordinate, $\theta$ is the polar angle at the end of $d s^{\prime}$, and $\alpha_{0}$ and $\theta_{0}$ are the pitch and polar angles, respectively, at $s_{0}$. By substituting (6) and (4) into (3) and integrating (numerically) over the polar angle in the interval $\left(\theta_{0}, \theta\right)$ we can calculate the energy of an electron in the plasmasphere as a function of $\theta$ and its initial energy and pitch angle.

The electron pitch angle at $\theta$ is given by the expression

$$
\begin{aligned}
\alpha\left(\theta, \theta_{0}, \alpha_{0}\right)= & \cos ^{-1}\left\{\frac { 1 } { \operatorname { s i n } ^ { 3 } \theta } \left[\sin ^{6} \theta\right.\right. \\
& \left.\left.-\sin ^{2} \alpha_{0} \sin ^{6} \theta_{0}\left(\frac{3 \cos ^{2} \theta+1}{3 \cos ^{2} \theta_{0}+1}\right)^{1 / 2}\right]^{1 / 2}\right\}
\end{aligned}
$$

The photoelectron flux along a field line in the plasmasphere can be easily calculated from the single particle energy and pitch angle, (3) and (5), and the magnetic field strength along the magnetic field line in question. For the present we are interested in obtaining an estimate of the total attenuation of the photoelectron flux in traversing the plasmasphere and the photoelectron flux buildup due to backscattering from the ionosphere below $1000 \mathrm{~km}$. Therefore we need only calculate the flux at the boundaries of the plasmasphere field tube connecting the local and conjugate ionospheres.

\section{Physical Parameters}

In the calculations we have employed two model atmospheres and ionospheres matched to the prevailing conditions on December 18, 1971, at Arecibo, Puerto Rico. For this day, plasma line intensity data were obtained with the Arecibo incoherent scatter radar. The intensity of the plasma line signal is related to the angular and energy spectra of the steady state photoelectron flux. These observations therefore provide a data base for direct comparison of calculated and measured plasma line intensities for matched solar, goemagnetic, and local thermospheric conditions. A detailed comparison of the observed and calculated plasma line intensities has been presented [Mantas et al., 1975] and will be further elaborated in a subsequent paper.

\section{Model Atmospheres and Ionospheres}

The model neutral atmospheres are based on the Jacchia [1971] model for the prevalent solar and geomagnetic conditions and are consistent with exospheric temperatures inferred from observed ion temperatures at $300 \mathrm{~km}$. The observed electron concentration profiles were matched to the plasma line altitudes (see, for example, Booker and Smith [1970], pp. 469,495 ) between 300 and $600 \mathrm{~km}$. The neutral atmospheric densities and the electron concentration profiles are given in Table 1 . We have taken the conjugate ionosphere and atmosphere to have the same density and composition as the ionosphere and atmosphere above Arecibo. This assumed symmetry simplifies the calculations considerably without affecting the conclusions reached in this study.

TABLE 1. Model Atmospheres and Electron Density Profiles

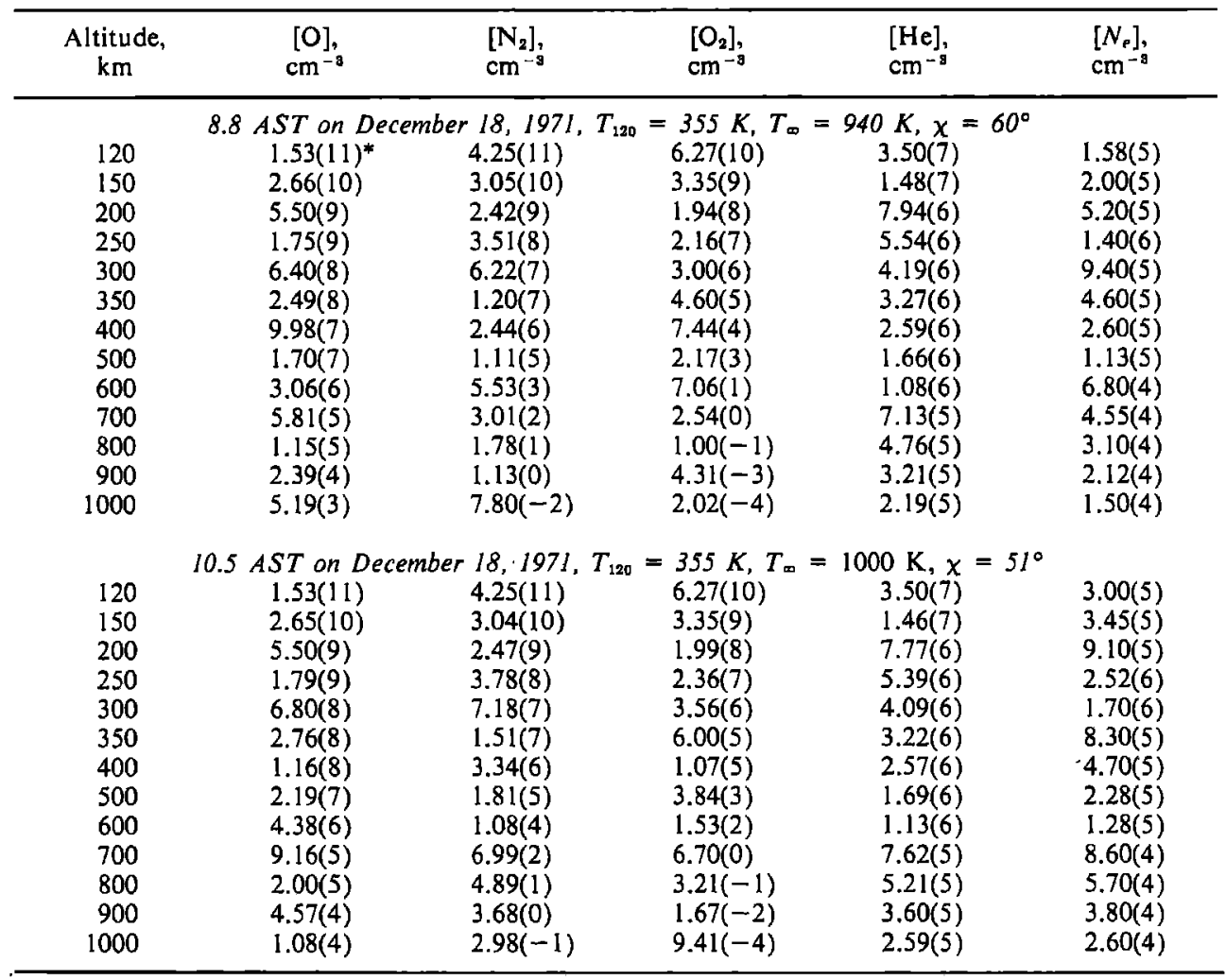

${ }^{*}$ Read $1.53(11)$ as $1.53 \times 10^{11}$. 
Plasma Distribution in the Plasmasphere

The electron density along a dipole magnetic field line in an isothermal $\left(T_{e}=T_{i}=3000^{\circ} \mathrm{K}\right)$ plasmasphere is given by [Angerami and Thomas, 1964]

$N_{e}(z)=\frac{N_{e 0}}{\eta^{1 / 2}} \exp \left(-z / H_{1}\right)+\eta_{2} \exp \left(-z / H_{2}\right)$

$$
\left.+\eta_{s} \exp \left(-z / H_{s}\right)\right\}^{1 / 2}
$$

where the geopotential height $z$ for an isothermal plasmasphere is

$$
\begin{aligned}
& z\left(\theta_{0}, \theta\right)=R_{0}\left\{\left(1-\sin ^{2} \theta_{0} / \sin ^{2} \theta\right)\right. \\
& \left.\quad+\frac{\Omega^{2} R_{0}}{2 g_{0}}\left(\sin ^{2} \theta_{0}-\sin ^{6} \theta / \sin ^{4} \theta_{0}\right)\right\}
\end{aligned}
$$

where $\theta_{0}$ is the polar angle of a reference point at $1000 \mathrm{~km}$, the foot of the magnetic field line connecting the local and conjugate ionospheres; $\theta$ is the polar angle of a point on the magnetic field line; $\Omega$ is the earth's angular velocity of rotation about its geographic axis; $H_{l}(i=1,2,3)$ are the scale heights for $\mathrm{O}^{+}, \mathrm{He}^{+}$, and $\mathrm{H}^{+}$, respectively, at the $1000 \mathrm{~km}$ reference altitude level; $\eta_{l}(i=1,2,3)$ are the ion densities at $1000 \mathrm{~km}$ relative to the $\mathrm{O}^{+}$density at that level; $\eta=\eta_{1}+\eta_{2}+\eta_{3} ; R_{0}$ is the geocentric distance of the reference level; $g_{0}$ is the earth's gravitational acceleration at $1000 \mathrm{~km}$; and $N_{e 0}$ is the electron density at $1000 \mathrm{~km}$. Here we extrapolate the observed $\mathrm{O}^{+}$to $1000 \mathrm{~km}$ and add light ion concentrations consistent with Arecibo observations from other days and with relevant satellite information [Breig and Hoffman, 1975].

\section{Cross Sections and Solar EUV Fluxes}

We have used the same set of collision cross sections as was used in previous photoelectron flux calculations reported by Mantas and Bowhill [1975] and Mantas and Walker [1976]. The parameters for representing the excitation cross sections by means of the Green and Barth [1965] empirical formula, as well as the parameters for representing the ionization cross sections with the Khare [1969] and Dalgarno and Lejeune [1971] formulas, can be found in the work of Mantas [1973] and Mantas and Walker [1976].

The primary photoelectron production rates were computed by using the neutral atmosphere models given in Table 1 , the solar EUV fluxes reported by Hinteregger [1970] and Hall and Hinteregger [1970], and the photoabsorption and photoionization cross sections given by Stolarski and Johnson [1972].

\section{Photoelectron Flux Pitch Angle and Energy Spectra in the Plasmasphere}

The angular distribution of the steady state photoelectron flux at $1000 \mathrm{~km}$ is given in Figure 1, where integrated spectra above a set of lower energy limits are shown. (The photoelectron distribution below $1000 \mathrm{~km}$ will be presented and compared with plasma line data in a subsequent communication.) These energies were chosen so that the calculated spectra can be compared directly with the measurements of Rao and Maier [1970]. The pitch angle is measured from the upward direction of the field line. Therefore electrons with pitch angles less than $90^{\circ}$ enter the plasmasphere while those with pitch angles greater than $90^{\circ}$ leave it. These spectra include contributions up through the second reflection. Figure la shows the pitch angle distribution of the photoelectron flux when both local

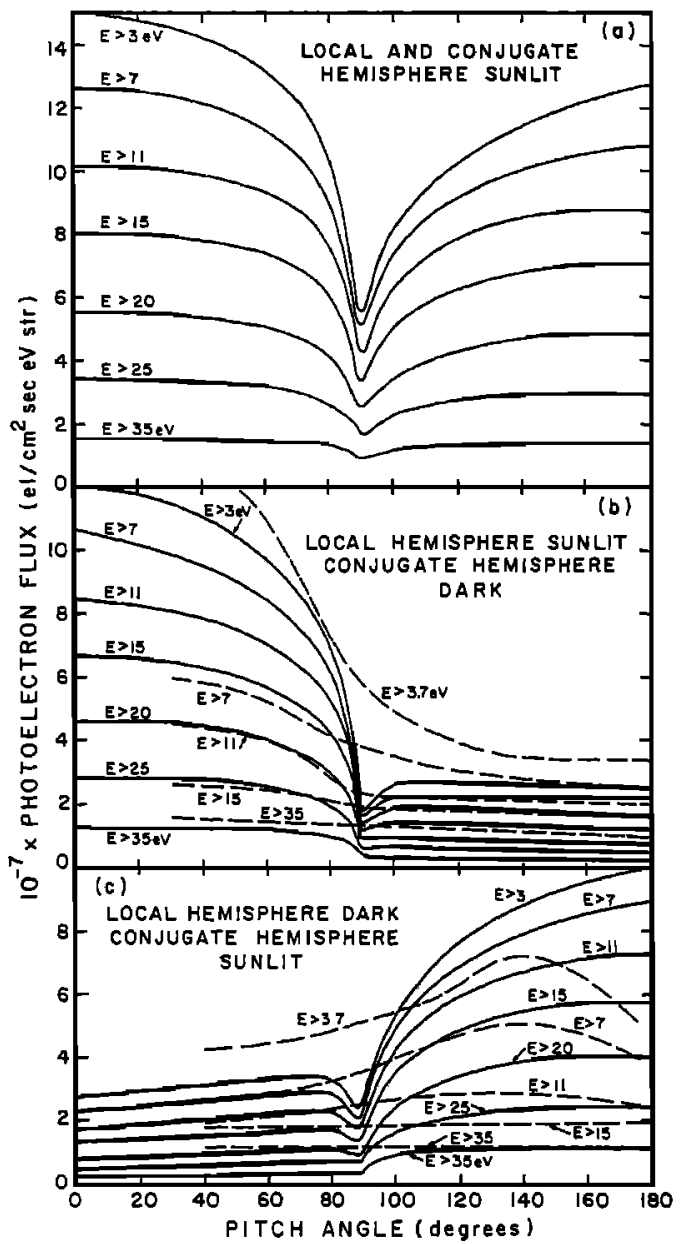

Fig. 1. Steady state photoelectron flux pitch angle spectra at 1000 $\mathrm{km}$ over Arecibo on December 18,1971, at $8.8 \mathrm{AST}$. The calculated spectra (solid curves) include contributions up to the second reflection of the escape flux between magnetically conjugate hemispheres. The measured spectra (dashed curves) have been taken from Figure 3 of Rao and Maier [1970]. The depletion around $90^{\circ}$ in the calculated spectra is due to neglecting Coulomb collisions in the plasmasphere.

and conjugate ionospheres are in sunlight, while Figures $2 b$ and $2 c$ (solid curves) show the distribution when the ionosphere is in sunlight only under the one end of the field line.

In Figures $1 b$ and $1 c$ we have also plotted (dashed curves) the angular spectra reported by Rao and Maier [1970]. These data were selected [Rao and Maier, 1970] from satellite Explorer 31 low-latitude $(1.2 \leq L \leq 2.5)$ passes at altitudes above $2300 \mathrm{~km}$, during the winter of 1965-1966. Therefore apart from covering the Arecibo magnetic latitude the measured spectra correspond to different altitudes and solar activity conditions than the calculated spectra. The comparison must therefore be restricted only to qualitative aspects, such as the overall shape and energy dependence; finer details are left for future comparisons. A rough qualitative agreement between the calculated and measured spectra is seen in the upwarddownward asymmetry. The calculated spectra show a minimum in the region around $90^{\circ}$ which is not present in the observed spectra. This minimum arises partly from the neglect of collisions in the plasmasphere; collisions would tend to redistribute the angular flux dependence, with the effect of 'filling up' the depleted region of the spectrum and diminishing the upward-downward flux anisotropy. The calculations agree with the observations that the backscattered flux component is 


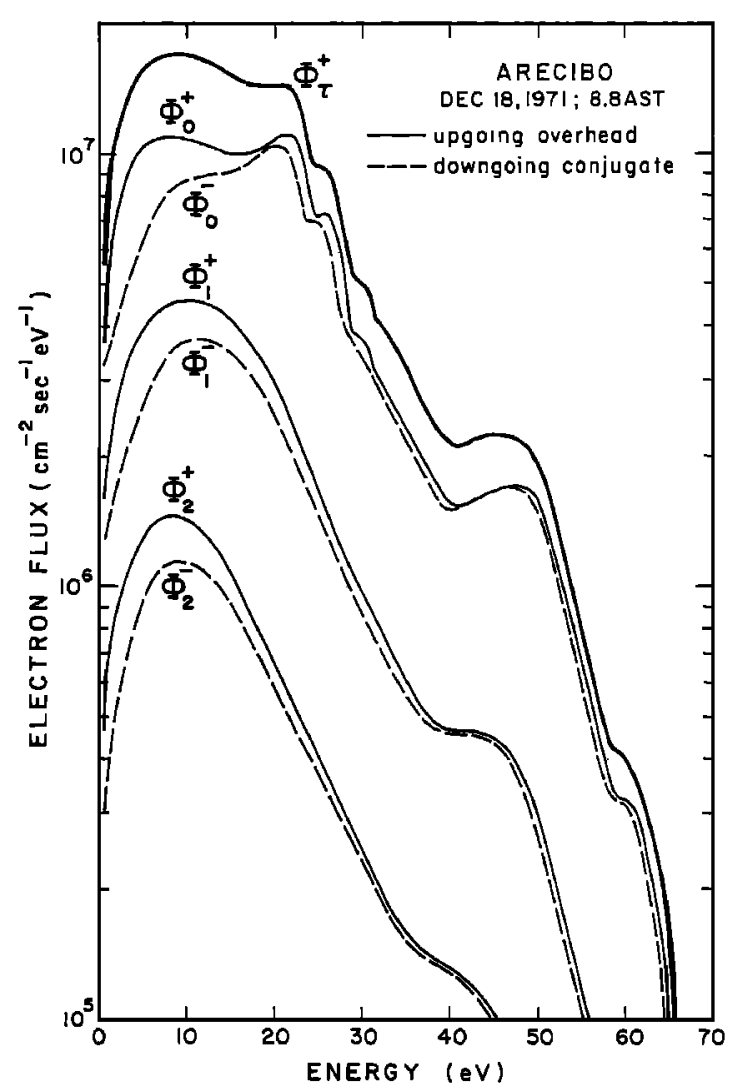

Fig. 2. Energy spectra of the escape photoelectron flux and of its two first reflections between local and conjugate hemispheres. The solid curves represent upward flux components at $1000 \mathrm{~km}$ over Arecibo. The dashed curves represent downward flux components at the other end of the magnetic field line at $1000 \mathrm{~km}$. The curve denoted $\Phi_{\tau}{ }^{+}$ represents the steady state upward flux at $1000 \mathrm{~km}$, when both local and conjugate hemispheres are sunlit.

more isotropic than the escaping component and also that the upward-downward flux anisotropy decreases with increasing electron energy.

The corresponding differential energy spectra are shown in Figure 2. The curves designated by $\Phi_{l}{ }^{+}(i=0,1,2)$ give the upward flux components ( $i$ indicates the number (order) of reflections (approximations)) at the 'foot' of the plasmaspheric field tube, while those designated by $\Phi_{i}-(i=0,1,2)$ give the components of the same order moving downward at the other 'end' of the magnetic field tube. The difference between two spectra of the same order (i.e., $\Phi_{l^{+}}-\Phi_{t^{-}}$) therefore represents the attenuation of the flux in traversing the plasmasphere. The curve indicated by $\Phi_{\tau}{ }^{+}$gives the steady state upward flux when both the local and conjugate ionospheres are in sunlight, obtained by superposition of all the upward contributions. The steady state downward flux can be obtained similarly. The steady state flux when only the ionosphere below the one end of the field line is sunlit can be obtained by superposition of the appropriate components. It should be noted here that the spectra shown in Figure 2 represent fluxes at the plasmasphere boundary in the inward and outward directions, projected along the magnetic field lines.

\section{Energy and Photoelectron Number Budgets in the Plasmasphere}

The energy and electron number budgets in a plasmaspheric field tube over Arecibo $(L=1.42)$ are summarized in Table 2.
The formula defining the entries in each column is shown at the top of that column. As was the case in Figure 2, the flux component order of reflection is given by the value of the subscript $(i=0,1,2)$, shown in the first column. In examining Table 2 the following scheme should be followed. The first entry,

$$
2 \pi \int_{0}^{E_{0}} \int_{0}^{1} \mu E \Phi_{0}\left(h_{0}, E, \mu\right) d \mu d E=6.07 \times 10^{9}\left(\mathrm{eV} / \mathrm{cm}^{2} \mathrm{~s}\right)
$$

is the energy flux entering the plasmasphere field tube boundary at $1000 \mathrm{~km}$ over Arecibo owing to photoelectron production in the ionosphere below. The third entry,

$$
2 \pi \int_{0}^{E_{0}} \int_{0}^{1} \mu E \Phi_{0}\left(h_{0}, E, \mu\right) d \mu d E=5.40 \times 10^{9}\left(\mathrm{eV} / \mathrm{cm}^{2} \mathrm{~s}\right)
$$

is that part of this energy flux which reaches the other end of the field line. The second entry, $6.67 \times 10^{8}$, is the difference between the two and represents the energy absorbed in the first passage of the escape flux through the plasmasphere. The second entry of the first column,

$$
2 \pi \int_{0}^{k_{0}} \int_{0}^{1} \mu E \Phi_{1}\left(h_{0}, E, \mu\right) d \mu d E=1.75 \times 10^{\circ}\left(\mathrm{eV} / \mathrm{cm}^{2} \mathrm{~s}\right)
$$

gives that part of the incident energy flux, $5.40 \times 10^{9}$ $\left(\mathrm{eV} / \mathrm{cm}^{2} \mathrm{~s}\right)$, backscattered from the ionosphere below. The following two entries, $2.50 \times 10^{8}$ and $1.50 \times 10^{\circ}$, are the fractions of the backscattered flux absorbed in and transmitted through the plasmasphere, respectively. The same scheme should be followed in the interpretation of the remaining entries appearing in this table. Also, the same procedure is to be followed in the electron number budget part of the table.

For the conditions modeled here, Table 2 shows that (1) $25-35 \%$ of the energy and $30-40 \%$ of the electron flux incident on the ionosphere are backscattered to the plasmasphere; (2) the transparency of the plasmasphere over Arecibo (collisions neglected) for energy transfer between conjugate hemispheres is $80-90 \%$, while for electron transfer it is between 70 and $85 \%$; (3) the steady state outgoing photoelectron flux at Arecibo is $5-10 \%$ higher than the escape flux when the conjugate ionosphere is in darkness and $30-40 \%$ higher when the conjugate ionosphere is sunlit.

It should be noted that the particle and energy fluxes given in Table 2 represent only particular moments of the photoelectron distribution and as such do not give a complete picture of the buildup effect. Perhaps a better illustration of the buildup effect is provided by a comparison of the number of photoelectrons, irrespective of their energy and direction of motion, contributed to a volume element by each successive reflection. The photoelectron number density is given by

$$
\rho_{t}(h)=2 \pi \int_{-1}^{1} \int_{0}^{E_{0}}\left\{\Phi_{l}(h, E, \mu) / v(E)\right\} d E d \mu
$$

The values of $\rho_{1}(i=0,1,2)$ at $1000 \mathrm{~km}$, and the sums over $i$ for local and conjugate sunlit and local sunlit conjugate dark conditions, relative to $\rho_{0}$, are given in Table 3 . This table shows that for the conditions considered here, about $40 \%$ of the photoelectrons found in a volume element at $1000 \mathrm{~km}$ over the observer, when the conjugate ionosphere is in darkness, consist of photoelectrons that have been backscattered from the conjugate ionosphere. When both the local and conjugate ionospheres are sunlit, more than half of the photoelectrons at $1000 \mathrm{~km}$ either are of conjugate origin or are photoelectrons of local origin that have been backscattered from the conjugate 
TABLE 2. Energy and Electron Number Budgets in the Plasmasphere

\begin{tabular}{|c|c|c|c|c|c|c|}
\hline \multirow{3}{*}{$\begin{array}{l}n_{0}=1000 \mathrm{~km} \\
E_{0}=100 \mathrm{ov}\end{array}$} & \multicolumn{3}{|c|}{ ENERGY BUDGET } & \multicolumn{3}{|c|}{ ELECTRON NUMAER GUDGET } \\
\hline & $\begin{array}{c}\text { UPWARD ENERGY } \\
\text { FLUX } \\
\left(\mathcal{O V V} / \mathrm{em}^{2} \mathrm{soc}\right) \\
2 \pi \int_{0}^{E_{0}} \int_{0}^{1} E \Phi_{1}\left(\mathrm{~h}_{0}, E, \mu\right) \mu d \mu d E\end{array}$ & $\begin{array}{c}\text { ENERGY ABSORBED } \\
\text { IN MAGNETOSPHERE } \\
\left(\bullet V / \mathrm{cm}^{2} \mathrm{soc}\right)\end{array}$ & $\begin{array}{c}\text { ENERGY FLUX } \\
\text { REACHING CONJUGATE } \\
\text { IONOSPHERE } \\
\left(e V / \mathrm{Cm}^{2} \mathrm{sec}\right) \\
2 \pi \int_{0}^{E_{0}} \int_{-1}^{o} E \Phi_{1}\left(h_{0}, E_{1} \mu\right)_{\mu} \mathrm{d} \mu \mathrm{d}\end{array}$ & $\begin{array}{c}\text { UPWARD ELECTRON } \\
\text { FLUX } \\
\left(e l / \mathrm{cm}^{2} \mathrm{sec}\right) \\
2 \pi \int_{0}^{\varepsilon_{0}} \int_{0}^{1} \Phi\left(h_{0}, \varepsilon_{,}, \mu\right) \mu d \mu \mathrm{dE}\end{array}$ & $\begin{array}{c}\text { ELECTRON FLUX } \\
\text { ABSORBED } \\
\text { IN MAGNETOSPHERE } \\
\text { (el/cm }\end{array}$ & $\begin{array}{c}\text { ELECTRON FLUX } \\
\text { REACHING CONJUGATE } \\
\text { IONOSPHERE } \\
\left(\mathrm{E} / \mathrm{cm}^{2} \mathrm{sec}\right) \\
2 \pi \int_{0}^{E_{0}} \int_{-1}^{0} \Phi\left(\mathrm{h}_{0}, E_{1} \mu\right) \mu d \mu d E\end{array}$ \\
\hline & & & ARECIBO DEC. & $.1971,88 \mathrm{AST}$ & & \\
\hline$\Phi_{0}^{ \pm}$ & $6.07 \times 10^{9}$ & $6.67 \times 10^{8}$ & $5.40 \times 10^{9}$ & $3.20 \times 10^{8}$ & $5.12 \times 10^{7}$ & $2.69 \times 10^{8}$ \\
\hline$\Phi_{1}^{ \pm}$ & $1.75 \times 10^{9}$ & $2.50 \times 10^{8}$ & $1.50 \times 10^{9}$ & $1.05 \times 10^{6}$ & $1.95 \times 10^{7}$ & $8.55 \times 10^{7}$ \\
\hline$\Phi_{2}^{ \pm}$ & $4.56 \times 10^{8}$ & $7.29 \times 10^{7}$ & $3.83 \times 10^{8}$ & $2.97 \times 10^{7}$ & $6.24 \times 10^{6}$ & $2.35 \times 10^{7}$ \\
\hline $\begin{array}{c}\Phi_{\text {totol }}^{ \pm} \\
\text {LOCAL COONUGATE } \\
\text { SUNLIT }\end{array}$ & $8.28 \times 10^{9}$ & $1.98 \times 10^{9}$ & $7.20 \times 10^{9}$ & $4.55 \times 10^{8}$ & $1.54 \times 10^{8}$ & $3.78 \times 10^{8}$ \\
\hline $\begin{array}{l}\text { \$iolal } \\
\text { LOCAL SULNIT }\end{array}$ & $6.53 \times 10^{3}$ & $9.90 \times 10^{8}$ & $5.78 \times 10^{9}$ & $3.50 \times 10^{8}$ & $7.69 \times 10^{7}$ & $2.93 \times 10^{9}$ \\
\hline & & & ARECIBO DEC. & 1971: 105 AST & & \\
\hline$\Phi_{0}^{ \pm}$ & $5.04 \times 10^{9}$ & $8.06 \times 10^{8}$ & $4.23 \times 10^{9}$ & $2.54 \times 10^{8}$ & $5.33 \times 10^{7}$ & $2.01 \times 10^{8}$ \\
\hline$\Phi_{1}^{ \pm}$ & $1.10 \times 10^{9}$ & $2.21 \times 10^{8}$ & $8.84 \times 10^{8}$ & $6.20 \times 10^{7}$ & $1.61 \times 10^{7}$ & $4.53 \times 10^{7}$ \\
\hline$\Phi_{2}^{*}$ & $2.31 \times 10^{8}$ & $5.07 \times 10^{7}$ & $1.80 \times 10^{8}$ & $1.34 \times 10^{7}$ & $4.01 \times 10^{6}$ & $9.36 \times 10^{6}$ \\
\hline $\begin{array}{l}\Phi_{\text {folol }}^{ \pm} \\
\text {LOCALCONUGATE } \\
\text { SUUNLIT }\end{array}$ & $6.37 \times 10^{9}$ & $2.16 \times 10^{9}$ & $5.29 \times 10^{9}$ & $3.29 \times 10^{8}$ & $1.47 \times 10^{8}$ & $2.56 \times 10^{8}$ \\
\hline $\begin{array}{l}\Phi_{\text {IOIOI }}^{2} \\
\text { LOCAL SUNLIT } \\
\text { CONJUGATE DARK }\end{array}$ & $5.27 \times 10^{9}$ & $1.08 \times 10^{9}$ & $4.41 \times 10^{9}$ & $2.67 \times 10^{8}$ & $7.34 \times 10^{7}$ & $2.10 \times 10^{8}$ \\
\hline
\end{tabular}

ionosphere. Since low-energy photoelectrons contribute most to the thermal electron gas heating, Table 3 implies that thermal electron gas heating rates at high altitudes are underestimated by more than a factor of 2 if photoelectrons from the conjugate ionosphere and collisional containment are neglected.

\section{Effect of Uncertainties in Plasmaspheric Electron Density and Ion Composition}

For the calculations shown in Figures 1-2 and Table 2 the relative ion concentrations at $1000 \mathrm{~km}$ were taken as $60 \% \mathrm{O}^{+}$, $15 \% \mathrm{He}^{+}$, and $25 \% \mathrm{H}^{+}$of the electron concentration. When data from the Isis 2 satellite [Breig and Hoffman, 1975] at 1400 $\mathrm{km}$ in winter 1971 are extrapolated to $1000 \mathrm{~km}$, they lead to relative ion concentrations of about $85 \% \mathrm{O}^{+}, 2 \% \mathrm{He}^{+}$, and $13 \%$ $\mathrm{H}^{+}$. For an isothermal plasma along the magnetic field line of $3000 \mathrm{~K}$ this ion composition at the boundary leads to a reduction of less than $5 \%$ in the total energy absorption rate in the plasmasphere and of less than $10 \%$ in the total electron number absorption rate. Changes in the plasma temperature of $\pm 1000 \mathrm{~K}$, for fixed electron density and ion composition at the boundaries, lead to changes of less than $2 \%$ and $5 \%$ in the total energy and electron number absorption rates, respectively. This is because the $\mathrm{H}^{+}$scale height is sufficiently large that reasonable variations in temperature change the $N_{e}$ content, along the field line here, by amounts of negligible consequence to the transmission. For the low $L$ value of Arecibo the fractional transmission above $1000 \mathrm{~km}$ should not vary significantly. The transmission can be expected to differ considerably, by $L$ values of 3 or more, between times of extended quiet and those, after a magnetic storm, of significantly depleted plasmaspheric content [e.g., Noxon and Johanson, 1970]. Thus at higher latitudes the flux buildup due to backscatter will tend to be less marked than is noted here; however, the flux buildup due to pitch angle diffusion in the plasmasphere will tend to increase.

\section{Transmission and BACKSCATTERING COEFFICIENTS}

The neglect of pitch angle scattering in the plasmasphere overestimates its transparency and therefore overestimates the buildup due to collisional backscatter containment. However, it underestimates the net buildup. This is because a photoelectron that undergoes pitch angle scattering in the plasmasphere is likely to be trapped there, while one reaching the ionosphere-plasmasphere boundary has a lower probability for containment through collisional backscattering from the lower ionosphere. In the steady state, pitch angle scattering in the plasmasphere would, of course, transfer a number of photoelectrons from nontrapped to trapped orbits, and vice versa; the net effect, nevertheless, is a flux buildup, since if pitch angle scattering is neglected, all trapped orbits will be unoccupied.

The magnitude of the buildup clearly depends on the transparency of the plasmasphere and on the magnitude of the fraction of the transmitted flux that is backscattered from the thermosphere. The transparency of the plasmasphere depends on the plasmasphere plasma content and on the energy and angular spectra of the photoelectron flux entering the plasmasphere. Similarly, the reflectivity of the thermosphere depends

TABLE 3. Fractional Buildup of Photoelectron Number Density at $1000 \mathrm{~km}$

\begin{tabular}{|c|c|c|}
\hline \multirow[b]{2}{*}{$i$} & \multicolumn{2}{|c|}{$\rho_{i} / \rho_{0}$} \\
\hline & 8.8 AST* $^{*}$ & 10.5 AST $\dagger$ \\
\hline $\begin{array}{l}0 \\
1 \\
2 \\
\text { Local and conjugate sunlit } \\
\text { Local sunlit, conjugate dark }\end{array}$ & $\begin{array}{l}1 \\
1.18 \\
0.43 \\
2.61 \\
1.43\end{array}$ & $\begin{array}{l}1 \\
1.05 \\
0.38 \\
2.43 \\
1.38\end{array}$ \\
\hline
\end{tabular}

From Arecibo, December 18, 1971.

$*_{\rho_{0}}=2.8 \mathrm{~cm}^{-\mathrm{s}}$.

$\dagger \rho_{0}=2.1 \mathrm{~cm}^{-1}$. 
on thermospheric conditions and the energy and angular spectra of the incident electron flux. Clearly, the steady state photoelectron flux in the plasmasphere will exhibit variations with plasmaspheric and thermospheric conditions. To estimate the relative importance to the flux enhancement of pitch angle diffusion versus backscatter, we examine in this section the magnitudes of the transmission and backscattering "coefficients' and their variation with ionospheric, plasmaspheric, and boundary conditions.

For the present purpose we will-define the coefficients for transmission of electrons, $T_{\Phi}$, and energy, $T_{\varepsilon}$, through the plasmasphere as the respective ratios of the total electron and energy fluxes exiting the plasmasphere, projected along the magnetic field line, to the corresponding total quantities entering the plasmasphere at the other end of the magnetic field line. In terms of the notation we have been using these can be written as

$$
\begin{gathered}
T_{\Phi} \equiv \frac{\int_{0}^{E_{0}} \int_{-1}^{0} \Phi\left(h_{0}, E, \mu\right) \mu d \mu d E}{\int_{0}^{E_{0}} \int_{0}^{1} \Phi\left(h_{0}, E, \mu\right) \mu d \mu d E} \\
T_{E} \equiv \frac{\int_{0}^{E_{0}} \int_{-1}^{2} E \Phi\left(h_{0}, E, \mu\right) \mu d \mu d E}{\int_{0}^{E_{0}} \int_{0}^{1} E \Phi\left(h_{0}, E, \mu\right) \mu d \mu d E}
\end{gathered}
$$

where the denominators are evaluated at the entry boundary and the numerators at the exit boundary.

Similarly, the coefficients for backscattering of electrons, $B_{\Phi}$, and energy, $B_{E}$, from the thermosphere will be defined as the respective ratios of the total upward electron and energy fluxes at $1000 \mathrm{~km}$, projected along the magnetic field line, to the corresponding downward quantities at the same altitude. Notationally, these coefficients are defined as

$$
\begin{aligned}
B_{\Phi} \equiv & \frac{\int_{0}^{k_{0}} \int_{0}^{1} \Phi\left(h_{0}, E, \mu\right) \mu d \mu d E}{\int_{0}^{E_{0}} \int_{-1}^{0} \Phi\left(h_{0}, E, \mu\right) \mu d \mu d E} \\
B_{E} \equiv & \frac{\int_{0}^{E_{0}} \int_{0}^{1} E \Phi\left(h_{0}, E, \mu\right) \mu d \mu d E}{\int_{0}^{E_{0}} \int_{-1}^{0} E \Phi\left(h_{0}, E, \mu\right) \mu d \mu d E}
\end{aligned}
$$

where the integrals are evaluated at the same boundary.

The plasmasphere transmission coefficients $T_{\Phi}$ and $T_{E}$ defined by $(7 a)-(7 b)$ can be obtained directly from Table 2 , as the ratios of the corresponding fluxes in the third and first columns in the same row. Similarly, the thermosphere backscattering coefficients $B_{\Phi}$ and $B_{E}$ can be obtained from Table 2, as the ratios of the corresponding fluxes in the first and third columns in two successive rows. It should be noted that the upward 'backscattered' flux may include contributions from secondary ionization. This allows the possibility that $B_{\Phi}>1$.

\section{Plasmasphere Transmission Coefficients}

It has been shown by Lejeune and Wormser [1976] that the transmission of electrons through the plasmasphere depends strongly on the value of a dimensionless parameter $C$ and that it is almost independent of the pitch angle distribution of the photoelectron flux incident upon the plasmasphere boundary. This parameter in the calculations of Lejeune and Wormser [1976] arises naturally in the reduction of the photoelectron transport equation to dimensionless form. Here we will derive $C$ by averaging (2) over the energy of the photoelectron flux incident upon the plasmasphere boundary and subsequently integrating the resulting expression:

$$
\begin{array}{r}
\left\langle\frac{d E^{+}}{d s}\right\rangle=\frac{2 \pi}{\Phi^{+}\left(h_{0}\right)} \int_{0}^{\infty} \int_{0}^{1}\left(\frac{d E}{d s}\right) \Phi\left(h_{0}, E, \mu\right) \mu d \mu d E \\
=-\beta \frac{N_{e}(s)}{\bar{E}^{+}}
\end{array}
$$

where the distance coordinate $s$, after the averaging over the pitch angle, is measured along the magnetic field line rather than along the particle trajectory, as is the case in (2) or (3). Integration of (9) leads to an expression similar to (3), which we will write here as

$$
\bar{E}^{+2}=2 \beta I(s)\{C(s)-1\}
$$

This gives the value of the mean energy $\bar{E}^{+}$of the incident electron flux at the distance $s$ from the plasmasphere boundary. The dimensionless parameter $C(s)=\left(\bar{E}_{0}{ }^{+}\right)^{2} /(2 \beta I(s))$ is seen to incorporate the mean energy $\bar{E}_{0}{ }^{+}$of the incident electron flux as well as the integrated plasma content $I(s) \equiv$ $\int_{s_{0}}^{8} N_{e}\left(s^{\prime}\right) d s^{\prime}$, both of which are important in determining the transmission of energetic electrons through the plasmasphere.

The values of $C$ (with the integral evaluated over the entire length of the magnetic field line in the plasmasphere), $\bar{E}_{0}{ }^{+}, T_{\Phi}$ and $T_{E}$ for each traversal $(i=0,1,2)$ of the plasmasphere, as well as for the superposed steady state fluxes, are given in Table 4. For comparison we have also included the transmission coefficients (for the symmetric case) calculated by Lejeune and Wormser [1976] with and without pitch angle scattering in the plasmasphere. In their calculations, Lejeune and Wormser

\begin{tabular}{|c|c|c|c|c|}
\hline$i$ & $\left(\bar{E}_{0}^{+}\right)_{l}$ & $C_{l}$ & $T_{\Phi_{l}}$ & $T_{E_{1}}$ \\
\hline \multicolumn{5}{|c|}{ Arecibo, $8.8 \mathrm{AST}$ on December $18,1971,2 \beta I=36.64 \mathrm{eV}^{2}$} \\
\hline-5 & 18.96 & 9.81 & 0.84 & 0.89 \\
\hline 1 & 16.64 & 7.56 & 0.81 & 0.86 \\
\hline 2 & 15.34 & 6.42 & 0.79 & 0.84 \\
\hline Local and conjugate sunlit & 18.20 & 9.04 & 0.83 & 0.88 \\
\hline Local sunlit, conjugate dark & 18.66 & 9.50 & 0.84 & 0.89 \\
\hline \multicolumn{5}{|c|}{ Arecibo, $10.5 \mathrm{AST}$ on December $18,1971,2 \beta I=63.44 \mathrm{eV}^{2}$} \\
\hline (1) & 19.84 & 6.20 & 0.79 & 0.84 \\
\hline 1 & 17.82 & 5.01 & 0.73 & 0.80 \\
\hline 2 & 16.52 & 4.30 & 0.70 & 0.78 \\
\hline Local and conjugate sunlit & 19.36 & 5.91 & 0.78 & 0.83 \\
\hline Local sunlit, conjugate dark & 19.74 & 6.14 & 0.79 & 0.84 \\
\hline \multicolumn{5}{|c|}{ Lejeune and Wormser [1976] } \\
\hline \multirow[t]{3}{*}{ No pitch angle diffusion } & 10.00 & 1.10 & 0.406 & 0.507 \\
\hline & 10.00 & 1.65 & 0.501 & 0.555 \\
\hline & 10.00 & 2.20 & 0.587 & 0.683 \\
\hline \multirow{3}{*}{$\begin{array}{l}\text { Local and conjugate sunlit, } \\
\text { symmetric }\end{array}$} & 10.00 & 1.10 & 0.25 & 0.31 \\
\hline & 10.00 & 1.65 & 0.32 & 0.38 \\
\hline & 10.00 & 2.20 & 0.39 & 0.46 \\
\hline
\end{tabular}
[1976] represented the energy spectrum of the electron flux entering the plasmasphere by the empirical formula

$$
\Phi^{+}\left(E, h_{0}\right) \sim E \exp \left[-\left(2 E / \bar{E}_{0}^{+}\right)\right]
$$

with $\bar{E}_{0}{ }^{+}=10 \mathrm{eV}$. For mean energies $\bar{E}_{0}{ }^{+}$in the range $15-20$

TABLE 4. Plasmasphere Photoelectron and Energy Transmission Coefficients 
$\mathrm{eV}$ this formula fits the energy distribution of the calculated upward fluxes at $1000 \mathrm{~km}$ reasonably well. However, the mean energy of $10 \mathrm{eV}$ chosen by Lejeune and Wormser [1976] is lower, by a factor in the range 1.5-2.0, than our calculated mean energy of the photoelectron flux entering the plasmasphere. The theoretically calculated mean energy is about 18-20 $\mathrm{eV}$ (see Table 4 and Mantas and Bowhill [1975]) and is in good agreement with the value (e.g., 16-18 eV) deduced from the Isis satellites photoelectron experiments [Wrenn and Heikkila, 1972; Wrenn, 1974].

Since the 'test' electron energy loss rate depends inversely on the electron energy (equation (2)), low-energy electrons are absorbed in a plasma more efficiently than electrons with higher energy. The softer electron energy spectrum assumed in the calculations of Lejeune and Wormser would therefore tend to underestimate the transparency of the plasmasphere to typical photoelectron fluxes. More important, it would tend to underestimate the effect (on the plasmasphere transmission) of pitch angle scattering relative to that of energy loss. This becomes clear when one considers that the ratio $\left(t_{D} / t_{E}\right)$ of the relaxation time for velocity dispersion $t_{D}$ to that for energy transfer $t_{E}$ [Spitzer, 1956, pp. 68-81], decreases as the energy of the nonthermal electron population increases.

These arguments can perhaps be better demonstrated by referring to Figure 3, where the plasmasphere transmission coefficients given in Table 4 are presented in graphical form, as a function of the parameter $C$. A reasonable extrapolation to lower $C$ values of the transmission coefficients calculated here is seen to lead naturally to the values obtained by Lejeune and Wormser [1976], with pitch angle diffusion neglected. High values of $C=\left(\bar{E}_{0}^{+}\right)^{2} / 2 \beta I$ represent conditions of either highenergy incident electron fluxes or low plasmasphere plasma content, while low values represent conditions of either lowenergy incident fluxes or high plasma content. The variation of the transmission coefficients, with pitch angle diffusion neglected, is seen to increase toward unity as $C \rightarrow \infty$ and to decrease toward zero as $C \rightarrow 0$, as is expected from physical considerations. Inclusion of pitch angle scattering reduces (Figure 3) the transmission coefficients to lower values than those obtained when diffusion is neglected.

From the three points calculated by Lejeune and Wormser it is not possible to determine the $C$ dependence of the transmission coefficients with pitch angle scattering included, except, of course, it is obvious that they should increase monotonically from zero toward unity as $C$ increases from zero to infinity. For typical escape photoelectron flux mean energies ( $15 \leq E \leq$
$20 \mathrm{eV}$ ) and the same plasmasphere plasma content as that used by Lejeune and Wormser the parameter $C$ takes values in the range $2.5 \leq C \leq 8.8$. A rough extrapolation of the photoelectron flux transmission coefficient $T_{\Phi}$, that includes pitch angle scattering, in this range of $C$ gives values roughly in the range $0.4 \leq T_{\Phi} \leq 0.7$. The important point to be noticed is that for typical photoelectron escape flux energy spectra and mid-latitude plasmasphere electron contents $(4 \leqslant C \leqslant 10)$ the photoelectron flux absorbed in the plasmasphere is about doubled when, in addition to energy loss, pitch angle scattering is included. For $C \leqslant 4$, on the other hand, pitch angle diffusion is of secondary importance to energy loss with regard to the transmission of energetic electrons through the plasmasphere. The soft electron spectrum chosen by Lejeune and Wormser [1976] is thus seen, on the one hand, to underestimate substantially the transparency of the plasmasphere to typical photoelectron fluxes for a given electron content and, on the other, to give an erroneous impression regarding the relative importance of energy loss to pitch angle scattering in determining the transmission of typical photoelectron fluxes through the plasmasphere.

\section{Thermosphere Backscattering Coefficients}

In the preceding section we have just seen that for midlatitudes, even though pitch angle scattering nearly doubles the photoelectron flux absorbed in the plasmasphere, more than half of the incident photoelectron flux is transmitted through the plasmasphere. This fraction is therefore still available for containment in the plasmasphere through collisional backscatter from the thermosphere below. It would be useful therefore to examine the variation of the backscattering coefficients with thermospheric and incident flux conditions.

Because of the host of processes that come into play in the interaction of an incident electron beam with the atmosphere it is not a priori obvious whether the variation of the backscattering coefficients can be described by a single dimensionless parameter (such as $C$ ) over a wide range of variation of thermospheric and incident flux conditions. However, we will find that it is useful to hypothesize that such a parameter, which we will call $X$, exists, at least for a limited range of variation of the thermospheric and incident flux conditions. Our search for this parameter will be guided by (1) examination of the most significant differences in thermospheric and boundary conditions for a number of cases where we have detailed calculations of backscattered electron and energy fluxes and (2) some general considerations of the backscattering problem.

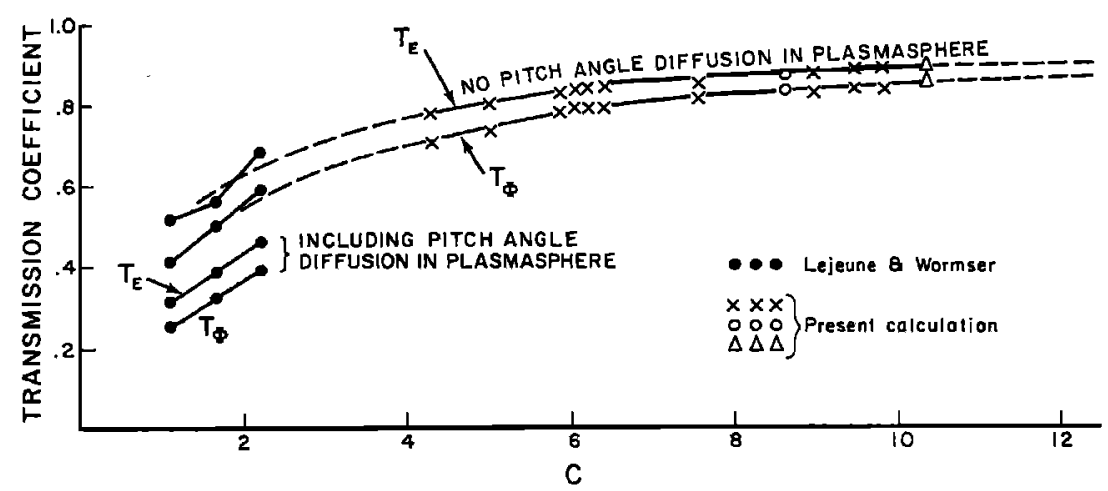

Fig. 3. Plasmasphere photoelectron $\left(T_{\Phi}\right)$ and energy $\left(T_{E}\right)$ transmission coefficients. For the definition of the parameter $C$ (abscissa), see the section on plasmasphere transmission coefficients. 
Examination of the two model calculations summarized in Table 2 and five additional model calculations (not otherwise discussed here), with nearly the same incident electron flux energy and angular spectra but varying thermospheric conditions, indicates that the topside $F$ region electron content plays a more important role relative to the other atmospheric constituents in influencing the backscattered to the incident flux ratio. This is not surprising when one considers that the incident electron flux must penetrate down to a relatively well defined neutral atmospheric pressure level before collisions increase substantially to deflect it and that the deflected flux contains a substantial number of energy-degraded primaries and (low energy) secondaries that are efficiently absorbed by the ambient thermal plasma. This implies that while the topside plasma content plays a secondary role, relative to the other constituents, in determining the depth of penetration and the probability for backscatter, it dominates the electron absorption rate along the upward path and thereby controls the backscattered to the incident electron flux ratio.

The calculations of Mantas and Walker [1976] show that for monoenergetic incident electron fluxes isotropically distributed in pitch angle in the downward direction and constant thermospheric conditions the ratio of the backscattered to the incident electron flux increases with increasing incident electron energy in the 25 - to $100-\mathrm{eV}$ range, while the corresponding energy flux ratio remains constant. The former of these results can be easily understood when one considers that as the incident electron energy increases, there are more secondaries produced and a wider spectrum of energy-degraded primaries that can escape. We will not digress to consider the latter result, since it does not influence the choice and combination of the thermospheric and boundary parameters in defining the dimensionless parameter we seek.

The above observations indicate that if the thermosphere backscattering coefficient for electrons, $B_{\Phi}$, is to increase with $X, X$ itself must increase with incident electron energy and decrease with topside $F$ region electron content. Consideration of the backscattering process itself suggests that $X$ must also depend on some suitably defined backscattering probability.

A dimensionless parameter that has these properties is the following:

$$
X \equiv A C^{\prime}(z) P(z)
$$

with $C^{\prime}(z)$ and $P(z)$ defined by

$$
\begin{aligned}
& C^{\prime}(z) \equiv\left(\bar{E}_{0}-\right)^{2}\left(2 \beta \int_{h_{0}}^{z} N_{e}(s) d s\right)^{-i} \\
& P(z) \equiv \frac{1}{2} \sum_{l}\left\langle\sigma_{l}^{e}(E)\right\rangle \int_{h_{0}}^{z} N_{l}(s) d s
\end{aligned}
$$

where $A$ is an arbitrary constant, $\bar{E}_{0}-$ is the mean energy of the electron flux incident upon the thermosphere at the boundary at $h_{0}$, and the other symbols have the same meaning as in (1) and (2), except that the elastic collision cross sections $\sigma_{l}{ }^{e}(E)$ have been averaged over the incident electron flux energy spectrum.

The integrals are evaluated along the magnetic field line from the boundary of the thermosphere at $h_{0}$ to some lower altitude $z$. The altitude $z$ will be specified as that giving the maximum distance $\left(h_{0}-z\right)$ that an incident electron with energy equal to the mean energy of the incident electron flux can penetrate into the thermosphere and still subsequently escape to the plasmasphere. To calculate $z$ we need an expression for the energy loss rate for an energetic electron in the ionosphere. An approximate expression for the energy loss rate of the same form as $(10)$ is

$$
\left\langle\frac{d E^{-}}{d s}\right\rangle=-\frac{1}{\bar{E}^{-}} \sum_{i}\left(\beta_{l}\right\rangle N_{l}(s)
$$

where $N_{l}(s)$ is the concentration of the $l$ th ionospheric constituent at the altitude $s$, and $\left\langle\beta_{l}\right\rangle$ are constants defined by

$$
\begin{aligned}
\left\langle\beta_{l}\right\rangle=\frac{1}{\Phi^{-}\left(h_{0}\right)} & {\left[\sum_{j} W_{l j} \int_{0}^{\infty} E \sigma_{l}{ }^{\prime}(E) \Phi^{-}\left(h_{0}, E\right) d E\right.} \\
+ & \left.\sum_{i}\left(W_{l l}+\bar{E}_{l l}\right) \int_{0}^{\infty} E \sigma_{l}{ }^{l}(E) \Phi^{-}\left(h_{0}, E\right) d E\right]
\end{aligned}
$$

where $\bar{E}_{l l}$ is the mean energy of the secondary electrons produced in the ionization of the $l$ th constituent, in the $i$ th ionic state. The rest of the symbols appearing in (13) have the same meaning as in (1). The constants $\left\langle\beta_{l}\right\rangle$ are seen to be related, within a factor $E$, to the stopping cross sections of the atmospheric constituents averaged over the incident electron flux energy spectrum.

Integration of (12) gives the energy of the electron along its trajectory. Since an electron must traverse the topside ionosphere at least twice (once downward and once upward) to escape, the maximum depth of penetration $\left(h_{0}-z\right)$ is obtained from the integrated form of (12) when the expression

$$
\frac{\left(\bar{E}_{0}^{-}\right)^{2}}{4}=\sum_{l}\left\langle\beta_{l}\right\rangle \int_{h_{0}}^{z} N_{l}(s) d s
$$

is satisfied. The solution of (14) is found by numerical or graphical means. Altitude $z$ having been specified, the parameter $X$ defined by (11) is specified within the scaling constant $A$; a convenient value for $A$ is $\frac{1}{2}$.

It must be noted that the parameter $X$ has been constrained only to exhibit the desired variation with incident flux mean energy and topside ionosphere electron content. It is otherwise arbitrary (i.e., several other parameters can be defined in terms of which the backscattered electron flux variation with boundary and thermospheric conditions can be represented). We have chosen $X$ from a number of other parameters on the criterion of giving the smallest standard deviation from a smooth curve when the calculated backscattered fluxes are plotted against each of these parameters.

Table 5 gives the values of $\bar{E}_{0}-, z, X, B_{\Phi}$, and $B_{E}$ for a number of thermosphere and incident flux conditions, including the two models used in the present study. The thermosphere backscattering coefficients $B_{\Phi}$ and $B_{E}$ have been plotted in Figure 4 against the parameter $X$. When the 'points' are

TABLE 5. Thermosphere Electron and Energy Backscattering Coefficients

\begin{tabular}{ccrcccc}
\hline $\bar{E}_{0}{ }^{-}, \mathrm{eV}$ & $z, \mathrm{~km}$ & $C^{\prime}(z)$ & $P(z)$ & \multicolumn{1}{c}{$X$} & $B_{\Phi}$ & $B_{E}$ \\
\hline 99.41 & 331.5 & 484.43 & 1.14 & 274.91 & 1.07 & 0.50 \\
49.46 & 362.0 & 130.96 & 0.89 & 58.11 & 0.87 & 0.49 \\
24.47 & 368.3 & 32.79 & 1.03 & 16.81 & 0.72 & 0.50 \\
20.89 & 345.0 & 6.88 & 0.72 & 2.46 & 0.32 & 0.26 \\
20.88 & 355.0 & 15.66 & 0.86 & 6.74 & 0.49 & 0.38 \\
20.77 & 364.5 & 6.54 & 0.71 & 2.32 & 0.31 & 0.26 \\
20.74 & 375.2 & 6.38 & 0.70 & 2.23 & 0.31 & 0.26 \\
20.52 & 354.9 & 6.48 & 0.72 & 2.33 & 0.30 & 0.25 \\
19.90 & 348.3 & 9.73 & 0.80 & 3.87 & 0.39 & 0.32 \\
19.81 & 330.8 & 9.91 & 0.81 & 3.99 & 0.40 & 0.32 \\
19.51 & 373.5 & 6.14 & 0.62 & 1.89 & 0.26 & 0.23 \\
17.30 & 352.5 & 7.60 & 0.77 & 2.91 & 0.35 & 0.30 \\
\hline
\end{tabular}




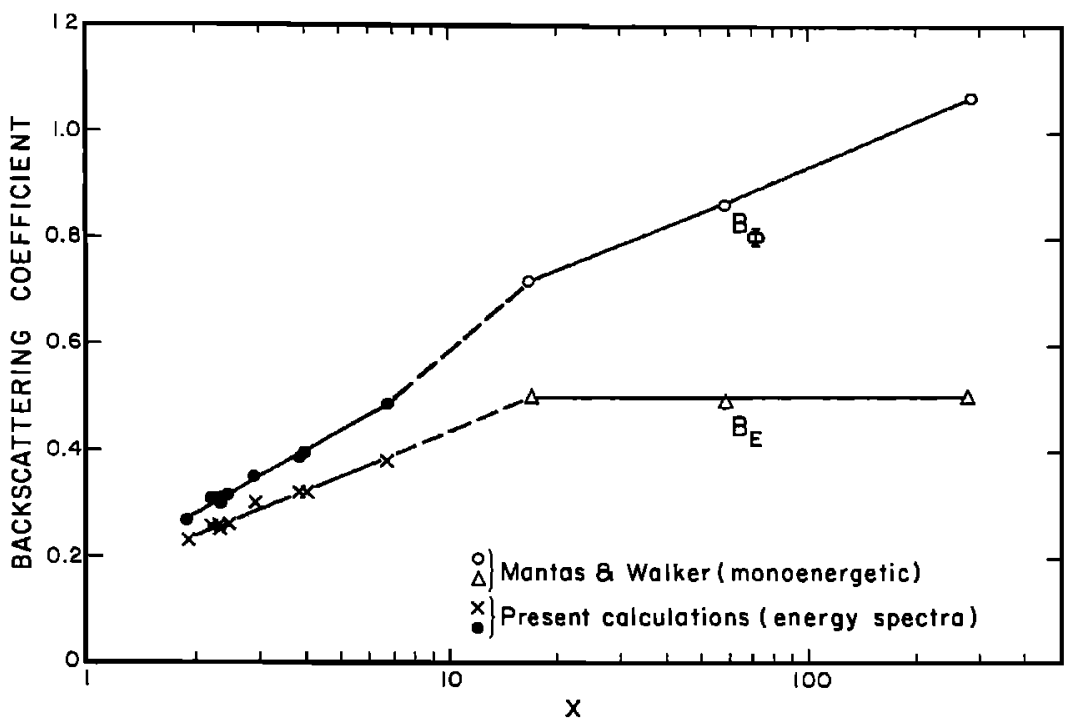

Fig. 4. Thermosphere photoelectron $\left(B_{\Phi}\right)$ and energy $\left(B_{E}\right)$ backscattering coefficients. For the definition of the parameter $X$ (abscissa), see the section on thermosphere backscattering coefficients. (The dashed curves have been inserted to aid the eye and to simplify the labeling. They should not be interpreted as extrapolations of the coefficients in this range of $X$.)

grouped in two sets, the one consisting of six points (three values of $B_{\Phi}$ and three of $B_{E}$ ) corresponding to the calculations of Mantas and Walker [1976] and the other to the remaining values given in Table 5 , it is seen that within each set $B_{\phi}$ and $B_{E}$ can be well represented as linear functions of $X$. Note that in the calculations of Mantas and Walker [1976] the incident electron fluxes were monoenergetic, while in the rest of the calculations the incident fluxes have broad energy spectra, e.g., those shown in Figure 2. The two different slopes obtained for the two types of incident electron flux energy spectra simply indicate that the thermosphere backscattering coefficients depend not only on the mean incident flux energy but also on the shape of the incident flux energy spectrum, hardly a surprising result.

The important point to be noted in Figure 4 is that the thermosphere backscattering coefficient for photoelectrons can be represented as a simple function of a single variable and that it can vary by as much as a factor of 2 , with typical variations of the thermospheric conditions, and probably varies more in exceptional cases.

\section{Estimated Photoelectron Fluxes Including Pitch ANgle Diffusion and Backscatter}

The transmission and backscattering coefficients, discussed in the previous section, can be used to estimate the amplitude of the steady state photoelectron flux in the plasmasphere, both backscattering from the thermosphere and pitch angle diffusion in the plasmasphere being included. We have already noted that the relatively soft electron spectrum employed by Lejeune and Wormser [1976] substantially underestimates the transparency of the middle latitude plasmasphere to typical photoelectron fluxes. For plasmasphere electron contents in the range $(0.7-2) \times 10^{13} \mathrm{~cm}^{-2}$ the plasmasphere transmission coefficient for photoelectrons, $T_{\Phi}$, lies roughly in the range $0.5-0.7$, energy loss and pitch angle diffusion being included. This estimate is in agreement with the value deduced by Wrenn [1974] from the Isis satellite photoelectron observations. The actual values of $T_{\Phi}$ must be found by solving the transport equation for photoelectrons in the plasmasphere-a task be- yond the purpose of the present paper. It is possible, however, even with the above rough estimate of the range of $T_{\phi}$, to obtain a fairly good estimate of the steady state photoelectron flux amplitude in the plasmasphere. The reason for this is that the contribution to the steady state flux by backscatter is in inverse relation to the contribution by pitch angle diffusion, in the sense that an increase in the contribution due to one mechanism leads to a decrease to the contribution due to the other, and vice versa. This compensating interaction tends to stabilize the steady state flux amplitude against small changes (or errors) in $T_{\Phi}$, making possible a fairly good estimate of the flux amplitude with only a rough knowledge of $T_{\Phi}$.

However, to calculate the steady state flux, in addition to the transmission and backscattering coefficients we must also know what fraction of the photoelectrons that undergo collisions in the plasmasphere is trapped and what fraction is backscattered to the thermosphere. The reason for this is clear. The trapped fraction can be counted to the steady state flux with little further consideration, while the backscattered (from the plasmasphere) fraction must be further considered, since it can still reenter the plasmasphere. In the present estimate we have assumed that all photoelectrons that undergo pitch angle scattering in the plasmasphere are trapped. This assumption considerably simplifies the algebra, in the calculation of the steady state flux, without leading to appreciable error. It is also supported by the analysis by Wrenn [1974] of satellite data. With this assumption the steady state photoelectron flux at the apex of the magnetic field tube is given by simple algebraic expressions in terms of the transmission and backscattering coefficients, the magnetic field tube geometry, and the firstorder approximation of the flux entering the field tube boundaries (see the appendix).

Table 6 gives the amplitude of the photoelectron flux $\Phi_{A}$ at the apex of the magnetic field tube with its foot over Arecibo ( $L=1.42$ ), various containment mechanisms being included, relative to the flux at the apex of the same tube, $\Phi_{a}{ }^{+}$, with no containment whatsoever. The plus sign indicates the direction away from Arecibo. When scattering is neglected, the flux at the tube apex, $\Phi_{a}{ }^{+}$, in terms of the flux entering the tube boundary at $1000 \mathrm{~km}, \Phi_{0}{ }^{+}$, is given by $\Phi_{a}{ }^{+}=T_{0 a}{ }^{e}\left(A_{0} / A_{a}\right) \Phi_{0}{ }^{+}$. 
TABLE 6. Calculated Photoelectron Fluxes at the Apex of the Magnetic Field Tube With. $L=1.42$, for Various Contajnment Conditions

\begin{tabular}{|c|c|c|c|c|}
\hline \multirow[b]{2}{*}{ Condition } & \multicolumn{2}{|c|}{$\begin{array}{l}\text { Local Sunlit, } \\
\text { Conjugate Dark }\end{array}$} & \multicolumn{2}{|c|}{$\begin{array}{c}\text { Local and } \\
\text { Conjugate Sunlit }\end{array}$} \\
\hline & $\Phi_{A}+/ \Phi_{a}+$ & $\Phi_{A}^{-} / \Phi_{a}^{+}$ & $\Phi_{A}^{+} / \Phi_{a}^{+}$ & $\Phi_{A}{ }^{-} / \Phi_{a}+$ \\
\hline \multicolumn{5}{|c|}{$8.8 \mathrm{AST}$ on December $18,1971, \Phi_{a}{ }^{+}=1.23 \times 10^{8} \mathrm{~cm}^{-2} \mathrm{~s}^{-1}$} \\
\hline No containment & 1.00 & 0 & 1.00 & 1.00 \\
\hline $\begin{array}{l}\text { Including pitch angle diffu- } \\
\text { sion but not backscatter }\end{array}$ & 1.10 & 0.10 & 1.19 & 1.19 \\
\hline $\begin{array}{l}\text { Including backscatter but } \\
\text { not pitch angle diffusion }\end{array}$ & 1.09 & 0.32 & 1.42 & 1.42 \\
\hline $\begin{array}{l}\text { Including backscatter and } \\
\text { pitch angle diffusion }\end{array}$ & 1.18 & 0.38 & 1.56 & 1.56 \\
\hline \multicolumn{5}{|c|}{ 10.5 AST on December 18.1971, $\Phi_{a}^{+}=9.58 \times 10^{7} \mathrm{~cm}^{-2} \mathrm{~s}^{-1}$} \\
\hline No containment & 1.00 & 0 & 1.00 & 1.00 \\
\hline $\begin{array}{l}\text { Including pitch angle diffu- } \\
\text { sion but not backscatter }\end{array}$ & 1.11 & 0.11 & 1.22 & 1.22 \\
\hline $\begin{array}{l}\text { Including backscatter but } \\
\text { not pitch angle diffusion }\end{array}$ & 1.05 & 0.24 & 1.29 & 1.29 \\
\hline $\begin{array}{l}\text { Including backscatter and } \\
\text { pitch angle diffusion }\end{array}$ & 1.16 & 0.32 & 1.48 & I. 48 \\
\hline
\end{tabular}

Here $A_{0}$ and $A_{a}$ are the cross-sectional areas of the tube at 1000 $\mathrm{km}$ and at the apex, respectively, and $T_{0 a}{ }^{e}$ represents the attenuation of the flux, due to energy loss alone, in its passage from $1000 \mathrm{~km}$ to the magnetic field tube apex. The values of $\Phi_{a}{ }^{+}$, for the two models considered here, are also given in Table 6.

For the nonsymmetric case when pitch angle diffusion in the plasmasphere and backscattering from the thermosphere are neglected, there is no return flux $\left(\Phi_{A}{ }^{-}=0\right)$ because there is no mechanism to produce a return flux. When pitch angle diffusion in the plasmasphere is included but backscattering from the thermosphere is neglected (as in the symmetric case in the calculations of Lejeune and Wormser [1976]), there is about a $10 \%$ enhancement in $\Phi_{A}{ }^{+}$. A return flux $\Phi_{A}{ }^{-}$equal to the enhancement in $\Phi_{A}{ }^{+}$is then also present. In the symmetric case the flux at the apex, in both directions, is enhanced by about $20 \%$ owing to pitch angle diffusion alone. When backscattering from the thermosphere is included but pitch angle diffusion in the plasmasphere is neglected, the enhancement of $\Phi_{A}{ }^{+}$is about $5-10 \%$. In this case the return flux is much larger, $25-30 \%$, than that due to pitch angle diffusion in the previous case. For symmetric conditions the enhancement in both directions, due to backscattering alone, is about $30-40 \%$; this is much larger than the enhancement due to pitch angle diffusion alone. When both pitch angle diffusion and backscatter are included, the enhancement of the flux, in both directions, is substantial in both the nonsymmetric and the symmetric case; in the symmetric case it is $48-56 \%$ for the models considered here. From the variation of the transmission and backscattering coefficients shown in Figures 3 and 4 it is clear that the buildup is strongly dependent on plasmaspheric and thermospheric conditions.

\section{Comparison With Experimental Results}

Photoelectron fluxes in the plasmasphere have been measured by Rao and Donley [1969], Maier and Rao [1970], Heikkila [1970], Rao and Maier [1970], Wrenn [1974], and others. These measurements have been carried out at different locations in the plasmasphere and pertain to a variety of geophysical and solar activity conditions. In comparing the available calculated and measured fluxes, one must therefore allow for the variation of the fluxes with location and geophysical and solar activity conditions. However, this variation is neither adequately understood nor often measured and reported. In such a comparison one is also faced with questions of instrumental effects and the handicap of nonuniform and/or inadequate definition of the reported quantities. All this substantially reduces the data base that can be unambiguously compared with theory. In addition, instrumental limitations confine the available photoelectron flux measurements to only that portion of the photoelectron energy spectrum above a certain lower energy limit. For the measurements included in the present comparison (see Table 7) the cutoff energies lie between 3.7 and $8 \mathrm{eV}$. From Figure 2 we see that about $10 \%$ of the upward steady state photoelectron flux, at $1000 \mathrm{~km}$, consists of photoelectrons with energies less than $5 \mathrm{eV}$, and about $25 \%$ of photoelectrons with energies less than $8 \mathrm{eV}$.

Table 7 summarizes selected photoelectron flux measurements in the plasmasphere. Of the available measurements we have included in this table only those pertaining to altitudes above $1000 \mathrm{~km}$, with $L \leqq 10$, and otherwise adequately defined to be reducible to fluxes parallel to the magnetic field lines (on the assumption of pitch angle isotropy at least within each directional hemisphere). The first part of the table includes the cases for which both ends (reference points) of the magnetic field line associated with the location of the measurement are sunlit, while the second includes those cases for which only one end is sunlit. The ends of the field line are taken at $300 \mathrm{~km}$, and an end is classified as sunlit when the solar zenith angle at this end is equal to or less than $100^{\circ}$.

The measured photoelectron fluxes in the plasmasphere are seen (Table 7) to lie in the range (1.4-3.5) $\times 10^{8} \mathrm{~cm}^{-2} \mathrm{~s}^{-1}$ when both ends of the field line are sunlit and $(0.15-1.5) \times 10^{8} \mathrm{~cm}^{-2}$ $\mathrm{s}^{-1}$ when only one end is sunlit. From Table 6 the calculated fluxes at the field tube apex are $\Phi_{A}=(1.42-1.92) \times 10^{\mathrm{a}}$ $\mathrm{cm}^{-2} \mathrm{~s}^{-1}$ for both ends of the line sunlit and $\Phi_{A}=(1.11-1.45)$ $\times 10^{8} \mathrm{~cm}^{-2} \mathrm{~s}^{1}$ for only the one end sunlit. These are seen to lie within the range of the measured flux values in both cases. It may even be noted that the calculated fluxes (corresponding to $L=1.42, h \simeq 2700 \mathrm{~km}$ ) agree more closely with the few nearby observations than with those further away. This may well merely be fortuitous, given that the models used in the calculations do not otherwise match the solar and geophysical conditions prevailing during these measurements.

Although the available reported measurements are not viewed as a data base adequate to provide any definitive conclusion regarding the range of flux variation, it is significant to note that when both ends of the field line are sunlit, the extreme measured fluxes are only a factor of 2.5 apart, while when only one end is sunlit, the measured fluxes span a range of an order of magnitude. Considering that the solar sunspot activity passed from a near minimum in 1965 to a near maximum in 1969 and considering the wide spread of altitudes $(1400-3500 \mathrm{~km}), L$ values $(1.2-10)$, and solar zenith angles encompassed in these measurements, the variation of the measured fluxes in the symmetric case is very small indeed. In the nonsymmetric case the variation is seen to be wider. Part of this is no doubt due to not distinguishing between measurements of fluxes in the direction toward versus away from the sunlit end of the field line, which are expected (see Table 6) to be substantially different in this case.

Though the following remarks are not necessary, they seem appropriate before concluding. It has been suggested [Cicerone et al., 1973; Cicerone, 1974] that comparisons of calculated 
TABLE 7. Measured Photoelectron Fluxes in the Plasmasphere

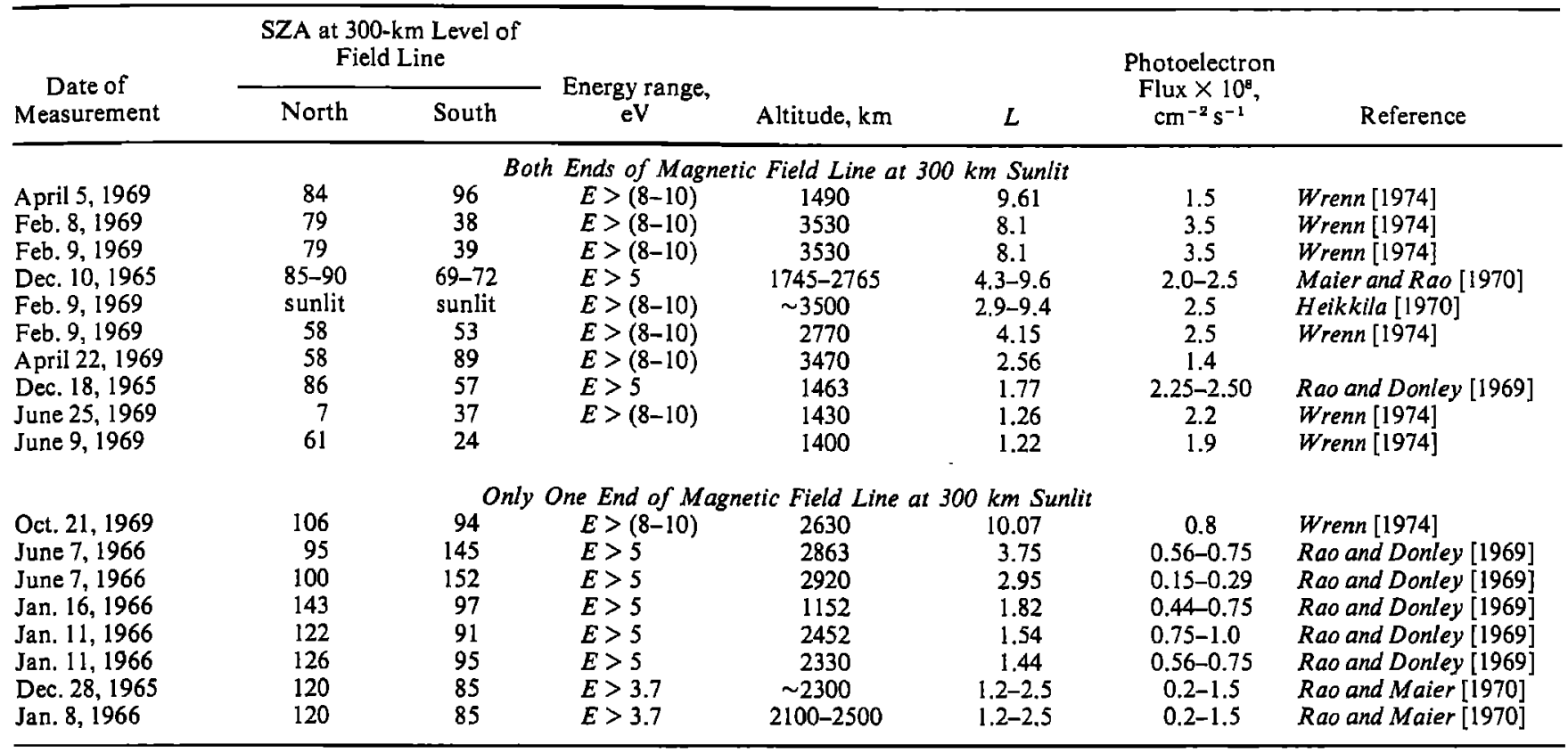

photoelectron escape fluxes with upward photoelectron fluxes at high altitudes deduced from plasma line intensity observations, and with in situ photoelectron flux measurements by satellite-borne probes, support the view [Roble and Dickinson, 1973; Swartz and Nisbet, 1973] that the solar EUV flux intensity measurements reported by Hinteregger [1970] underestimate the true solar EUV flux intensity by about a factor of 2.

Before arguing for or against the accuracy of the solar EUV flux measurements, one must keep in mind that the energy flux carried by the upward photoelectron flux above, say, $600 \mathrm{~km}$ (about $(1-10) \times 10^{9} \mathrm{eV} \mathrm{cm}^{-2} \mathrm{~s}^{-1}$ ) constitutes less than $1 \%$ of the energy flux (about $1.5 \times 10^{12} \mathrm{eV} \mathrm{cm}^{-2} \mathrm{~s}^{-1}$ ) in the relevant wave length range (about 1025-10 $\AA$ ) of the solar EUV, and therefore the former constitutes one of the least favorable criteria upon which the magnitude of the latter can be tested. This statement, of course, requires further qualification, since the precise knowledge of the escape photoelectron flux, and the associated energy, would allow one to use this quantity as a criterion, whether it is favorable or not. Let us state here without proof (the reader can verify this statement by consulting Cicerone et al. [1973], Cicerone [1974], and references cited in these papers) that the measured and calculated photoelectron fluxes on the basis of which the accuracy of the solar EUV flux intensity measurements has been questioned do not have the required accuracy to warrant the conclusion that these measurements underestimate the true solar EUV flux intensity.

\section{SUMMARY}

It has been known for some time that a substantial fraction of a low-energy electron flux incident on the top of the atmosphere is backscattered to the plasmasphere. While photoelectrons at $F$ region altitudes lose their energy through a variety of elastic and inelastic processes, the only effective energy sink for photoelectrons confined in the plasmasphere is the ambient thermal electron gas. For energies greater than about $10 \mathrm{eV}$ this is a slow process, and, as a result, photoelectrons confined at plasmasphere heights have much longer lifetimes than photoelectrons confined at lower altitudes. This implies that processes which tend to confine photoelectrons to plasmaspheric altitudes, such as collisional backscattering of photoelectrons from the thermosphere and magnetic trapping due to pitch angle redistribution of the photoelectron flux through Coulomb collisions in the plasmasphere, increase the steady state photoelectron flux in the plasmasphere above that which would have been attained in the absence of containment.

The transmission of photoelectrons through the plasmasphere and their backscattering from the thermosphere can be described in a simple manner in terms of the photoelectron flux spectral characteristics and the properties of the plasmasphere and the thermosphere. The transparency of the plasmasphere is reduced substantially when pitch angle diffusion in the plasmasphere is included in the calculations. However, the transmission coefficient of the mid-latitude plasmasphere, for our photoelectron fluxes, is significantly larger than that calculated by Lejeune and Wormser [1976]. The coefficient for backscattering of photoelectrons from the thermosphere increases with the mean energy of the incident electron flux spectrum and decreases with increasing topside $F$ region plasma content. For typical photoelectron energy spectra and typical variations of the topside $F$ region plasma content the thermosphere backscattering coefficient can vary by as much as a factor of 2 or more in exceptional cases.

The plasmasphere transmission and the thermosphere backscattering coefficients can be used to obtain fairly accurate estimates of the steady state photoelectron flux amplitude in the plasmasphere and thermosphere, with the effects of pitch angle diffusion and backscatter included. Such estimates have been presented for the steady state flux at the apex of representative $L=1.4$ (Arecibo) magnetic field tubes. These calculations have shown that the steady state flux in the plasmasphere is substantially enhanced by collisional and magnetic containment of photoelectrons to plasmaspheric heights. The calculated fluxes are found to lie within the range of measured photoelectron fluxes in the plasmasphere. A critical examination of the calculated and experimental high-altitude photoelectron fluxes thought to support the view that the measured solar EUV flux [Hinteregger, 1970] has been underestimated 
reveals that these fluxes do not have the required accuracy to warrant such a conclusion.

It has also been noted that the usual separation of photoelectron flux calculations in the thermosphere from those in the plasmasphere must be avoided in favor of a calculation of the photoelectron distribution along the entire length of the magnetic field tube, above, say, $120 \mathrm{~km}$. This common incision-a cesarean of expediency rather than necessity-has proliferated a terminology (as: escape flux, plasmasphere 'transparency,' thermosphere 'reflectivity,' 'transmission and reflection coeffcients,' electron albedo, etc.) which conceals the essential continuity of the photoelectron distribution along the entire length of the magnetic field lines and by now constitutes a hindrance rather than an aid to the proper conceptualization of the physical problem. While it is productive to consider separately the processes leading to these terms, when the results of such investigations are applied to the actual problem, they must be tempered by a proper appreciation of the relevance of the entire medium within the magnetic field tube to the properties of the photoelectron distribution.

\section{APPENDIX}

Let $\Phi_{o l^{+}}$and $\Phi_{o c}{ }^{+}$be the upward photoelectron fluxes at $1000 \mathrm{~km}$ over the local and conjugate ionosphere, respectively, obtained by solving the transport equation in the region below $1000 \mathrm{~km}$, with the boundary condition of no incident flux at $1000 \mathrm{~km}$ and the assumption that photoelectrons reaching this altitude from below are free to escape. Denote by $i$ the number of traversals of the plasmasphere, $T_{i}^{e}$ the plasmasphere transmission coefficient for photoelectrons when pitch angle diffusion in the plasmasphere is neglected, $T_{t a}{ }^{e}$ the plasmasphere transmission coefficient from the boundary to the apex of the field line when pitch angle diffusion in the plasmasphere is neglected, $T_{i}$ the plasmasphere transmission coefficient when both pitch angle diffusion in the plasmasphere and energy loss to the ambient thermal electron gas are included, $D_{t} \equiv T_{t}{ }^{e}-$ $T_{i}$, and $B_{i}$ the thermosphere backscattering coefficient for photoelectrons. Then the steady state photoelectron flux amplitude $\Phi_{A}^{ \pm}$, over the entire cross-sectional area of the magnetic field tube at the apex, for a variety of conditions, under the assumptions of the section on estimating steady state photoelectron fluxes, can be estimated by the following simple algebraic expressions.

In the case when the ionosphere under one end, say, the local end, of the magnetic field tube is sunlit and that under the other is in darkness we can write

No containment whatsoever

$$
\begin{aligned}
& \Phi_{A}{ }^{+}=T_{0 a}{ }^{e} \Phi_{0 l}{ }^{+} \\
& \Phi_{A^{-}}=0
\end{aligned}
$$

The plus sign indicates the direction away from the observer.

Pitch angle diffusion in the plasmasphere neglected, collisional backscatter from the thermosphere included

$$
\begin{aligned}
& \Phi_{A^{+}}=\left\{T_{0 a}{ }^{e}+T_{2 a}{ }^{e} B_{2} T_{1}{ }^{e} T_{0}{ }^{e}\right\} \Phi_{0 l}{ }^{+} \\
& \Phi_{A^{-}}=\left\{T_{1 a}{ }^{e} B_{1} T_{0}{ }^{e}\right\} \Phi_{0 l}{ }^{+}
\end{aligned}
$$

Pitch angle diffusion in the plasmasphere included, collisional backscatter from the thermosphere neglected

$$
\begin{aligned}
& \Phi_{A^{+}}=\left\{T_{0 a}^{e}+\frac{1}{2} D_{0}\right\} \Phi_{0 l^{+}} \\
& \Phi_{A^{-}}=\frac{1}{2} D_{0} \Phi_{0 l^{+}}
\end{aligned}
$$

Both pitch angle diffusion in the plasmasphere and collisional backscatter from the thermosphere included

$$
\Phi_{A}^{ \pm}=\Phi_{c}^{ \pm}+\Phi_{d} \pm
$$

where

$$
\begin{aligned}
& \Phi_{c}^{+}=\left\{T_{0 a}^{e}+T_{2 a}{ }^{e} B_{2} T_{1} B_{1} T_{0}\right\} \Phi_{0 l}^{+} \\
& \Phi_{c}{ }^{-}=\left\{T_{1 a}{ }^{e} B_{1} T_{0}\right\} \Phi_{0 l}{ }^{+} \\
& \Phi_{d}{ }^{+}=\Phi_{d}^{-}=\frac{1}{2}\left\{D_{0}+D_{1} B_{1} T_{0}+D_{2} B_{2} T_{1} T_{2} T_{0}\right\} \Phi_{0 l}{ }^{+}
\end{aligned}
$$

In the case when both the local and conjugate ionospheres are sunlit we can write

No containment whatsoever

$$
\begin{aligned}
& \Phi_{A^{+}}=T_{0 a}^{e} \Phi_{0 t^{+}} \\
& \Phi_{A^{-}}=T_{0 a}{ }^{e} \Phi_{0 c}{ }^{+}
\end{aligned}
$$

Pitch angle diffusion in the plasmasphere neglected, collisional backscatter from the thermosphere included

$$
\begin{aligned}
& \Phi_{A}{ }^{+}=\left\{T_{0 a}{ }^{e}+T_{2 a}{ }^{e} B_{2} T_{1}{ }^{e} T_{0}{ }^{e}\right\} \Phi_{0 l}{ }^{+}+\left\{T_{1 a}{ }^{e} B_{1} T_{0}{ }^{e}\right\} \Phi_{0 c}{ }^{+} \\
& \Phi_{A}{ }^{-}=\left\{T_{1 a}{ }^{e} B_{1} T_{0}{ }^{e}\right\} \Phi_{0 l}{ }^{+}+\left\{T_{0 a}{ }^{e}+T_{2 a}{ }^{e} B_{2} T_{1}{ }^{e} T_{0}{ }^{e}\right\} \Phi_{0 c}{ }^{+}
\end{aligned}
$$

The expressions for the remaining cases can be written similarly. In the case for which symmetry is assumed $\left(\Phi_{0 l}{ }^{+}=\right.$ $\Phi_{o c}{ }^{+}$) and the coefficients of the same order (i.e., the same value of the index $l$ ) are equal, the above expressions can be further simplified by combining various terms.

Acknowledgments. This work was supported by the National Science Foundation under grant DES 74-03082-A03 to the University of Texas at Dallas and grant ATM 72-01644 to the Stanford Research Institute.

The Editor thanks E. G. Fontheim and W. E. Swartz for their assistance in evaluating this paper.

\section{REFERENCES}

Angerami, J. J., and J. O. Thomas, Studies of planetary atmospheres, 1, The distribution of electrons and ions in the earth's exosphere, $J$. Geophys. Res., 69, 4537, 1964.

Bailey, G. J., R. J. Moffett, and W. E. Swartz, Effects of photoelectron heating and interhemisphere transport on day-time plasma temperatures at low latitudes, Planet. Space Sci., 23, 599, 1975.

Banks, P. M., and A. F. Nagy, Concerning the influence of elastic scattering upon photoelectron transport and escape, $J$. Geophys. Res., 75, 1902, 1970.

Bauer, P., G. Lejeune, and M. Petit, Thermal coupling between the upper $F_{2}$-region and the magnetosphere: Heat fluxes and energy production, Planet. Space Sci., 18, 1447, 1970.

Booker, H. G., and E. K. Smith, Comparative study of ionospheric measurement techniques, J. Atmos. Terr. Phys., 32, 467, 1970.

Breig, E. L., and J. H. Hoffman, Variations in ion composition at middle and low latitudes from Isis 2 satellite, J. Geophys. Res., 80, 2207, 1975.

Carlson, H. C., Jr., Ionospheric heating by magnetic conjugate point photoelectrons, J. Geophys. Res., 71, 195, 1966.

Carlson, H. C., Preliminary observations of $6300 \AA$ predawn enhancement at Arecibo, Aurora and Airglow, edited by B. M. McCormac, Reinhold, New York, 1967.

Carlson, H. C., Survey of satellite and ground based measurements of photoelectron excited emissions, Ann. Geophys., 30, 59, 1974.

Carlson, H. C., and G. M. Weill, Solar cycle variation of conjugate photoelectron flux onset timing deduced from $6300 \AA$ and $T_{e}$ observations, Ann. Geophys., 23, 569, 1967.

Cicerone, R. J., Photoelectrons in the ionosphere: Radar measurements and theoretical computations, Rev. Geophys. Space Phys., 12, 259,1974

Cicerone, R. J., and S. A. Bowhill, Photoelectron fluxes measured at Millstone Hill, Radio Sci., 6, 957, 1971a.

Cicerone, R. J., and S. A. Bowhill, Photoelectron fluxes in the ionosphere computed by a Monte Carlo method, J. Geophys. Res., 76, $8299,1971 b$. 
Cicerone, R. J., W. E. Swartz, R. S. Stolarski, A. F. Nagy, and J. S. Nisbet, Thermalization and transport of photoelectrons: A comparison of theoretical approaches, J. Geophys. Res., 78, 6709, 1973.

Dalgarno, A., and G. Lejeune, The absorption of electrons in atomic oxygen, Planet. Space Sci., 19, 1653, 1971.

Doering, J. P., W. G. Fastie, and P. D. Feldman, Photoelectron excitation of $\mathrm{N}_{2}$ in the day airglow, J. Geophys. Res., 75, 4787, 1970.

Doering, J. P., W. K. Peterson, C. O. Bostrom, and T. A. Potemra High resolution daytime photoelectron energy spectra from AE-E, Geophys. Res. Lett., 3, 129, 1976.

Evans, J. V., Midlatitude electron and ion temperatures at sunspot minimum, Planet. Space Sci., 15, 1557, 1967a.

Evans, J. V., Electron temperature and ion composition in the $F_{1}$ region, J. Geophys. Res., 72, 3343, $1967 b$.

Evans, J. V., The heating of the protonosphere, Space Res, VIII, 717, 1968.

Evans, J. V., and G. P. Mantas, Thermal structure of the temperate latitude ionosphere, J. Atmos. Terr. Phys., 30. 563, 1968.

Fontheim, E. G., A. E. Beutler, and A. F. Nagy, Theoretical calculations of the conjugate predawn effects, $A$ nn. Geophys., 24, 489, 1968.

Geisler, J. E., and S. A. Bowhill; lonospheric temperatures at sunspot minimum, J. Atmos. Terr. Phys., 27, 457, $1965 a$.

Geisler, J. E., and S. A. Bowhill, Exchange of energy between the ionosphere and the protonosphere, J. Atmos. Terr. Phys., 27, 1119, $1965 b$.

Green, A. E. S., and C. A. Barth, Semiempirical cross sections for electron impact, J. Geophys. Res., 72, 3933, 1965.

Hagen, J. B., and P. Y. S. Hsu, The structure of the protonosphere above Arecibo, J. Geophys. Res., 79, 4269, 1974.

Hall, L. A., and H. E. Hinteregger, Solar radiation in the extreme ultraviolet and its variation with solar rotation, J. Geophys. Res., 75 , $6959,1970$.

Hanson, W. B., Electron temperatures in the upper atmosphere, Space Res., III, 282, 1963.

Hays, P. B., and W. E. Sharp, Twilight airglow, 1, Photoelectrons and [O I] 5577-A radiation, J. Geophys. Res., 78, 1153, 1973.

Heikkila, W. J., Photoelectron escape flux observations at mid-latitudes, J. Geophys. Res., 75, 4877, 1970.

Hinteregger, H. E., The extreme ultraviolet solar spectrum and its variation during a solar cycle, $A n n$. Geophys., 26, 547, 1970.

Jacchia, L. G., Revised static models of the thermosphere and exosphere with empirical temperature profiles, Spec. Rep. 332, Smithson. Astrophys. Observ., Cambridge, Mass., 1971.

Khare, S. P., Energy spectrum of the secondary electrons and the fluorescent efficiency of electrons in the $3914 \AA$ band, Planet. Space Sci. 17, 1257, 1969.

Knudsen, W. C., Latitudinal variation of differential photoelectron flux at 560-km altitude, J. Geophys. Res., 77, 1233, 1972.

Lejeune, G., and F. Wormser, Diffusion of photoelectrons along a field line inside the plasmasphere, J. Geophys. Res., 81, 2900, 1976.

Maier, E. J., and B. C. N. Rao, Observations of the suprathermal electron flux and the electron temperature at high latitudes, J. Geophys. Res., 75, 7168, 1970.

Manson, S. T., D. J. Kennedy, A. F. Starace, and D. Dill, Angular distribution of photoelectrons from atomic oxygen, nitrogen and carbon, Planet. Space Sci., 22, 1535, 1974.

Mantas, G. P., Electron collision processes in the ionosphere, Ph.D. thesis, Univ. of Ill., Urbana, 1973.

Mantas, G. P., Theory of photoelectron thermalization and transport in the ionosphere, Planet. Space Sci., 23, 337, 1975.

Mantas, G. P., and S. A. Bowhill, Calculated photoelectron pitch angle and energy spectra, Planet. Space Sci., 23, 355, 1975.

Mantas, G. P., and J. C. G. Walker, The penetration of soft electrons into the ionosphere, Planet. Space Sci., 24, 409, 1976.

Mantas, G. P., V. B. Wickwar, and H. C. Carlson, Plasma line and theoretical studies of photoelectrons at Arecibo (abstract), Eos Trans. AGU, 56, 1037, 1975.

Mariani, F., Pitch angle distribution of the photoelectrons and the origin of the geomagnetic anomaly in the $F_{2}$ layer, J. Geophys. Res. 69. 556, 1964

Nagy, A. F., and P. M. Banks, Photoelectron fluxes in the ionosphere, J. Geophys. Res., 75, 6260, 1970.

Nagy, A. F., and P. M. Banks, Photoelectrons and related ionospheric effects, Space Res., XI, 1123, 1971.

Nagy, A. F., J. D. Winningham, and P. M. Banks, The effect of conjugate photoelectron impact ionization on the pre-dawn ionosphere, J. Atmos. Terr. Phys., 35, 2289, 1973.

Nisbet, J. S., Photoelectron escape from the ionosphere, J. Atmos. Terr. Phys., 30, 1257, 1968.

Noxon, J. F., and A. E. Johanson, Effect of magnetically conjugate photoelectrons on OI(6300\&), Planet. Space Sci., 18, 1367, 1970.

Petit, M., Mesures de températures, de densité électronique et de composition ionique dans l'ionosphère par diffusion de Thomson: Etude déséquilibre thermodynamique dans l'ionosphère diurne, Ann. Geophys., 24, 1, 1968.

Rao, B. C. N., and J. L. Donley, Photoelectron flux in the topside ionosphere measured by retarding potential analyzers, J. Geophys. Res., 74, 1715, 1969.

Rao, B. C. N., and E. J. R. Maier, Photoelectron flux and protonospheric heating during the conjugate point sunrise, $J$. Geophys. Res., 75, 816, 1970.

Roble, R. G., and R. E. Dickinson, Is there enough solar extreme ultraviolet radiation to maintain the global mean thermospheric temperature?, J. Geophys. Res., 78, 249, 1973.

Sanatani, S., and W. B. Hanson, Plasma temperatures in the magnetosphere, J. Geophys. Res., 75, 769, 1970.

Schunk, R. W., and P. B. Hays, Photoelectron energy losses to thermal electrons, Planet. Space Sci., 19, 113, 1971.

Shawhan, S. D., L. P. Block, and C. G. Falthammar, Conjugate photoelectron impact ionization, J. Atmos. Terr. Phys., 32, 1885 , 1970.

Spitzer, L., Physics of Fully Ionized Gases, Interscience Tracts on Physics and Astronomy, no. 3, pp. 68-81, Interscience, New York, 1956.

Stolarski, R. S., and N. P. Johnson, Photoionization and photoabsorption cross sections for ionospheric calculations, J. Atmos. Terr. Phys., 34, 1691, 1972.

Swartz, W. E., and J. S. Nisbet, Incompatibility of solar EUV fluxes and incoherent scatter measurements at Arecibo, J. Geophys. Res. $78,5640,1973$.

Swartz, W. E., G. J. Bailey, and R. J. Moffett, Electron heating resulting from interhemispherical transport of photoelectrons, Planet. Space. Sci.. 23, 589, 1975.

Wickwar, V. B., The $6300 \AA$ predawn enhancement: Excitation by photoelectrons from the magnetic conjugate point, Ann. Geophys., 28, 187, 1972 .

Wickwar, V. B., Conjugate phatoelectrons at $L=5.6$ and the $6300 \AA$ postsunset enhancement, Planet. Space Sci., 22, 1297, 1974.

Wormser, F., Diffusion des photoélectrons dans la magnétosphère, Ph.D. thesis, Univ. of Paris VII, Paris, 1973.

Wrenn, G. L., Satellite measurements of photoelectron fluxes, $A n n$. Geophys., 30, 49, 1974.

Wrenn, G. L., and W. J. Heikkila, Photoelectron measurements with Isis 1 and 2 satellites (abstract), Eos Trans. AGU, 53, 474, 1972.

Yngvesson, K. O., and F. W. Perkins, Radar Thomson scatter studies of photoelectrons in the ionosphere and Landau damping, $J$. Geophys. Res., 73, 97, 1968.

(Received June 30, 1976; accepted July 22, 1977.) 